\title{
Dark matter and dark forces from a supersymmetric hidden sector
}

\author{
S. Andreas, ${ }^{*}$ M. D. Goodsell, ${ }^{\dagger}$ and A. Ringwald ${ }^{*}$ \\ Deutsches Elektronen-Synchrotron, DESY, Notkestraße 85, 22607 Hamburg, Germany
}

(Received 8 February 2012; published 3 January 2013)

\begin{abstract}
We show that supersymmetric "dark force" models with gravity mediation are viable. To this end, we analyze a simple string-inspired supersymmetric hidden sector model that interacts with the visible sector via kinetic mixing of a light Abelian gauge boson with the hypercharge. We include all induced interactions with the visible sector such as neutralino mass mixing and the Higgs portal term. We perform a detailed parameter space scan comparing the produced dark matter relic abundance and direct-detection cross sections to current experiments.
\end{abstract}

DOI: $10.1103 /$ PhysRevD.87.025007

PACS numbers: 12.60.Jv, 12.60.Cn, 14.70.Pw, 95.35.+d

\section{INTRODUCTION}

There has been much interest recently in the possibility that there exists a hidden sector containing a dark matter particle coupled to a hidden $\mathrm{U}(1)$ gauge boson (a dark force) having a mass of the order of a $\mathrm{GeV}$ that kinetically mixes with the photon [1-5]. Such a scenario could explain many astrophysical puzzles, such as the positron excess observed by PAMELA [6], ATIC [7], and Fermi [8], or the direct-detection and annual modulation signals of DAMA [9], CoGeNT [10,11] and CRESST [12] (if one ignores the disputed $[13,14]$ contradiction due to XENON100 [15] and CDMS [16]). Following from the work of Refs. [17-19], elegantly simple supersymmetric models in the latter category were constructed in Ref. [20] and further examined in Ref. [21] (see also Ref. [22]). However, these works emphasized that, in order to obtain such a light hidden sector, supersymmetry breaking effects in the visible sector would necessarily be dominated by gauge mediation, in order that the masses should be acceptably small. Thus it is natural to ask whether confirmation of these observations would be in contradiction with gravity mediation-in other words, whether it is also possible to have a gravity-mediated spectrum of particles that can yield similar phenomenology. This is also linked to the interesting question as to whether these models can be embedded into string theory: such hidden sectors appear very naturally there-see, e.g., Refs. [23-31]—but the problem of finding gauge mediation dominance over gravity mediation is notoriously difficult to achieve in globally consistent models.

Beyond the dark matter motivation, it is also useful to ask what hidden sector models of this form coming from string theory are allowed or excluded by current observations. This is because, even if the hidden sector does not comprise (all) the dark matter, there is a wealth of experiments capable of probing dark forces over a very wide

\footnotetext{
*sarah.andreas@desy.de

† mark.goodsell@desy.de mark.goodsell@cern.ch

†andreas.ringwald@desy.de
}

range of hidden gauge boson mass and kinetic mixing values. Kinetic mixing was considered in the context of the heterotic string in Refs. [26,32-35]. It has been examined in type II strings in Refs. [23-25,36-40]; in Refs. [25,29], both masses and mixings were considered, and it was argued that the dark forces scenario could be accommodated provided that there is additional sequestering. In this work, we shall consider hidden sector models with the particle content and similar couplings to those in Ref. [20], but argue that when we have gravity mediation domination, these can still give interesting phenomenological predictions under certain mild assumptions, without requiring additional sequestering relative to the visible sector. Although we will discuss the possible explanation of the signals found by DAMA and CoGeNT, these will therefore not be our primary motivation: rather, we wish to explore how simple supersymmetric hidden dark sectors with a hidden $\mathrm{U}(1)$ can be constrained by observations.

The paper is organized as follows. In Sec. II, we describe the model of a supersymmetric dark sector that we shall be examining. This is followed by a summary of constraints upon hidden U(1)s with hidden matter charged under them in Sec. III. There we also include the reach of future fixed target experiments and illustrate these with an investigation of a simple toy model. Section IV then contains the meat of the paper: the results of the parameter search over our supersymmetric dark sector model. We include additional technical details in the Appendix: the hidden sector renormalization group equations (RGEs) in Appendix A, the spectrum of the model in Appendix B (including the mass mixing matrix with the visible neutralino in $B$ 2), a review of kinetic and mass mixing of a massive hidden gauge boson with the hypercharge and $Z$ in Appendix $C$, and a description of the Goldstone boson mixing in Appendix D. In addition, in Appendix E, we discuss the supersymmetryinduced Higgs portal term and the mixing of the hidden and minimal supersymmetric standard model (MSSM) Higgs fields; we believe that although the existence of the term has been known in the literature (see, e.g., Ref. [41] in nonsupersymmetric models and Ref. [42] in the supersymmetric 
context), the effect of the mixing terms for direct detection have not been given elsewhere. Included is a calculation of the induced coupling of the hidden dark matter Majorana fermion to nucleons.

\section{SUPERSYMMETRIC DARK SECTORS}

\section{A. Supersymmetric kinetic mixing}

We shall consider models that interact with the visible sector primarily through kinetic mixing of a hidden U(1) gauge field with the hypercharge. Hence, we have a holomorphic kinetic mixing $\chi_{h}$ between hypercharge $B_{\alpha}$ with coupling $g_{Y}$ (and gaugino the Bino, $b$ ) and hidden gauge superfield $X_{\alpha}$ with coupling $g_{h}$ (and gaugino written as $\lambda$ ) appearing in the Lagrangian density

$$
\mathcal{L} \supset \int d^{2} \theta\left(\frac{1}{4 g_{Y}^{2}} B^{\alpha} B_{\alpha}+\frac{1}{4 g_{h}^{2}} X^{\alpha} X_{\alpha}-\frac{\chi_{h}}{2} B^{\alpha} X_{\alpha}\right) .
$$

The physical kinetic mixing in the canonical basis [25,39] is then given by

$$
\chi=g_{Y} g_{h} \operatorname{Re}\left(\chi_{h}\right) .
$$

We shall assume no matter charged under both hidden and visible gauge groups, so this relationship is valid at all energy scales. Since we are considering string-inspired models with a "hidden" $\mathrm{U}(1)$, that is, without matter charged under both the visible and hidden gauge groups, we shall take the value of the holomorphic kinetic mixing parameter to be of the order of a loop factor [25]:

$$
\chi_{h} \equiv \frac{\kappa}{16 \pi^{2}} .
$$

Here, $\kappa$ is a number that must, in principle, be derived from the high-energy model; in a field theory model, it is generated by integrating out some heavy linking fields (charged under visible and hidden sectors) at one loop, whereas in string models, it can be understood as arising from Kaluza-Klein modes of closed strings. In all cases, it depends only logarithmically upon mass splittings of the spectrum, and we shall therefore either take it to be equal to one or to vary by at most an order of magnitude from unity $[25,28,29] .{ }^{1}$ We thus have

$$
\chi=g_{Y} g_{h} \frac{\kappa}{16 \pi^{2}}
$$

the most commonly taken value for $\chi$ is thus of the order of $10^{-3}$, but smaller values correspond to decreasing the hidden gauge coupling which may be extremely small in the case of hyperweak groups $[25,29,44]$. Henceforth, we shall always use the physical mixing $\chi$.

\footnotetext{
${ }^{1}$ Our results only depend on the absolute value of the mixing parameter. Effects that are sensitive to the different signs have been studied in Ref. [43].
}

As befits a well-studied subject, there are a variety of notations. In addition to using $\chi$, we shall also adopt the notation used in Ref. [45]: ${ }^{2}$

$$
\begin{gathered}
\chi \equiv-\sin \epsilon \equiv-s_{\epsilon} \quad \cos \epsilon \equiv c_{\epsilon} \equiv \sqrt{1-\chi^{2}}, \\
\tan \epsilon \equiv t_{\epsilon}=-\frac{\chi}{\sqrt{1-\chi^{2}}} .
\end{gathered}
$$

However, a crucial novelty in this work is the application of relation (2.4) to parameter scans rather than allowing for independent $\chi$ and $g_{h}$, which we shall see in Sec. III D will lead to qualitatively different results for the cross sections.

\section{B. Hidden matter fields}

The model that we shall consider is the simplest possible without adding dimensionful supersymmetric quantities. There are three chiral superfields $S, H_{+}, H_{-}$with $H_{+}$and $H_{-}$charged under the hidden $\mathrm{U}(1)$ with charges \pm 1 . These appear in a superpotential with dimensionless coupling $\lambda_{S}$

$$
W \supset \lambda_{S} S H_{+} H_{-} \text {. }
$$

This is inspired by $D$-brane models where the singlet is essentially the adjoint of the gauge group: the superpotential above arises due to the $N=2$-like structure, and there is no renormalizable singlet potential due to this; alternatively, there may be $N=2$ supersymmetry of the couplings at some scale, although we shall not enforce this. Such hidden sectors from string theory were considered in, e.g., Refs. [27,31], and the above model was studied with gauge mediation in Ref. [20] where it was termed a "hidden sector next-to-minimal supersymmetric standard model (NMSSM)," although we have set the cubic singlet term in the superpotential to zero. There then exists a global U(1) symmetry under which $S$ and $H_{-}$are charged; string theory will not respect this, and we consider that it shall either be broken at higher order in the superpotential or through nonperturbative effects-but we shall assure that it will play no role in the following.

Once we include soft supersymmetry-breaking terms, we have the approximate potential for the hidden sector,

$$
\begin{aligned}
V= & \left|\lambda_{S}\right|^{2}\left(\left|S H_{+}\right|^{2}+\left|S H_{-}\right|^{2}+\left|H_{+} H_{-}\right|^{2}\right)+\frac{g_{h}^{2}}{2}\left(\left|H_{+}\right|^{2}\right. \\
& \left.-\left|H_{-}\right|^{2}-\xi\right)^{2}+m_{+}^{2}\left|H_{+}\right|^{2}+m_{-}^{2}\left|H_{-}\right|^{2} \\
& +m_{S}^{2}|S|^{2}+\left(\lambda_{S} A_{S} S H_{+} H_{-}+\frac{1}{2} M_{\lambda} \lambda \lambda+\text { c.c. }\right), \quad(2.7)
\end{aligned}
$$

where $\xi=-\frac{\chi}{g_{h}} \xi_{Y}=\chi\left(g_{Y} / g_{h}\right) g_{Y} \frac{v^{2}}{4} \cos 2 \beta$. The approximation lies in the $D$-term potential; the full form is found in Appendix E.

\footnotetext{
${ }^{2}$ Note, however, that this differs from the expressions in Ref. [20], which defines $\chi=-\tilde{\epsilon} \equiv-t_{\tilde{\epsilon}}, s_{\tilde{\epsilon}} \equiv-\frac{\chi}{\sqrt{1-\chi^{2}}}, c_{\tilde{\epsilon}} \equiv$ $\frac{1}{\sqrt{1-\chi^{2}}}$, although there they write $\epsilon$ instead of $\tilde{\epsilon}$ (we added the tilde to avoid confusion with the above). On the other hand, Refs. [43,46-50] define $\delta \equiv-\chi$.
} 

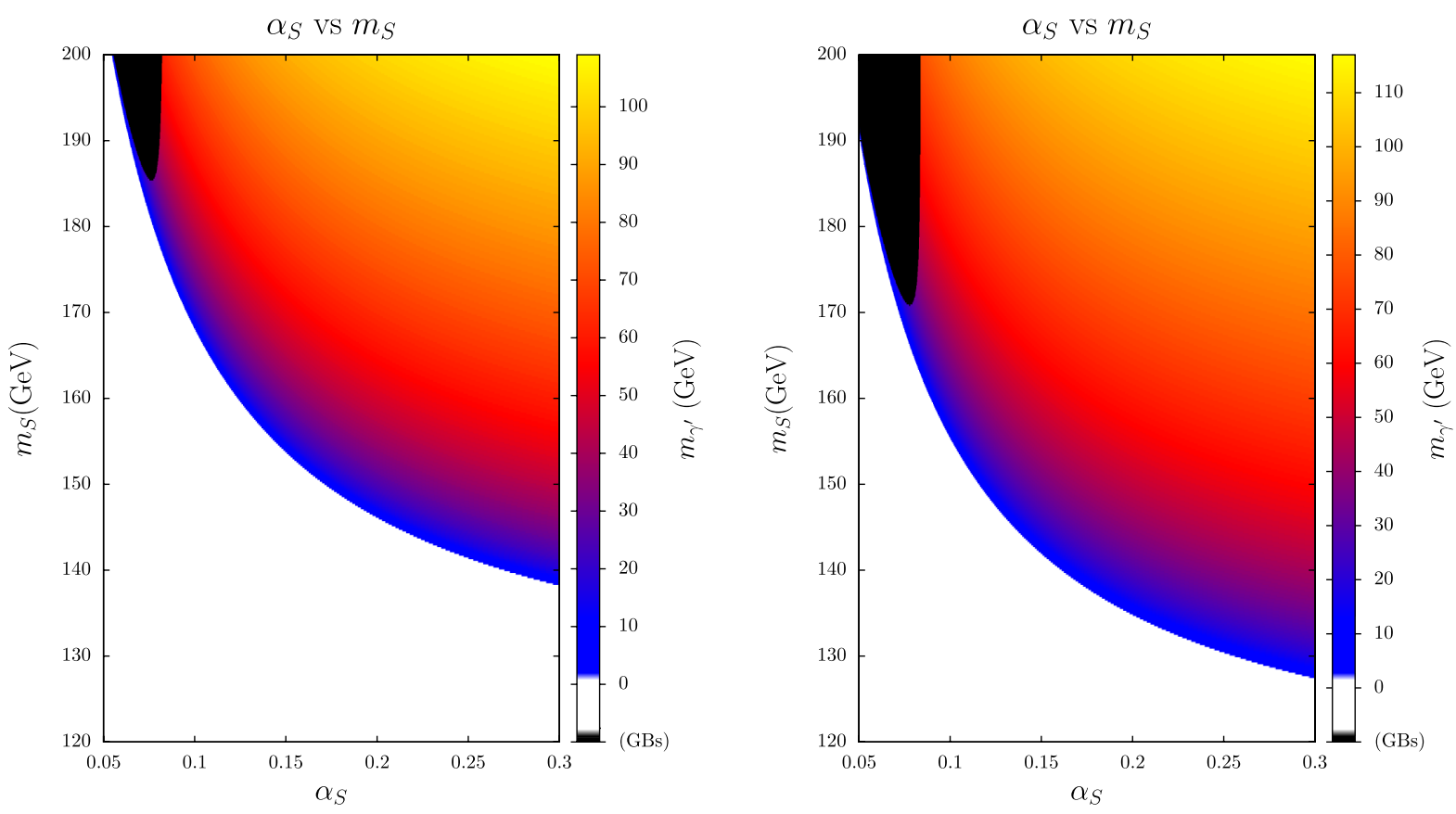

FIG. 1 (color online). Hidden photon mass $m_{\gamma^{\prime}}$ induced by radiative hidden gauge symmetry breaking, scanned over $m_{S}$ and $\alpha_{S} \equiv$ $\frac{\lambda_{S}^{2}}{4 \pi}$. In both, $m_{H}=A_{S}=100 \mathrm{GeV}, \alpha_{h}=0.0417$. Left: $M_{\lambda}=71 \mathrm{GeV}$, right: $M_{\lambda}=50 \mathrm{GeV}$. All values given at $10^{16} \mathrm{GeV}$. The black region shows no stable symmetry breaking.

A crucial difference for the phenomenology of the model once we consider gravity mediation is, however, that the gravitino is not the lightest supersymmetric particle (LSP), and therefore the dark matter can consist of stable hidden sector particles. We can thus perform a full analysis of the model, including the visible sector and its couplings, using micrOMEGAs [51-55] to determine the relic abundance and direct-detection cross sections.

\section{Symmetry breaking through running}

Just as in the MSSM, the top Yukawa coupling can, through running from the grand unified theory scale, induce electroweak symmetry breaking, so in the model we are considering, the Yukawa coupling $\lambda_{S}$ can induce breaking of the hidden gauge symmetry. By choosing the soft masses and couplings at the MSSM grand unified theory scale, we can then find models at the low-energy scale with hidden gauge symmetry breaking. A priori the independent supersymmetric parameters are $\chi, g_{h}, \lambda_{S}$ and the soft masses $m_{H_{ \pm}}, m_{S}, A_{S}$ and $M_{\lambda}$ (the hidden gaugino mass) which we can choose at the high-energy scale and run down.

Via Eq. (2.4), we are asserting a relation between $\chi$ and $g_{h}$. Thus, if we take $\kappa=1$, we reduce the number of free parameters in the model by one. However, as described above, we shall in certain plots (Figs. 3 and 5 right, 6 right, 7 and 8 right, 9 and 10 right and 11) allow an order of magnitude variation in $\kappa$; hence, although this does not strictly reduce the number of parameters in the model, it does rather constrain them with important consequences. Finally, we shall make one further assumption about the parameters: we shall take $m_{H_{+}}=m_{H_{-}}$at the high-energy scale. This is motivated by the fields $H_{ \pm}$being a nonchiral pair [note that we are taking no explicit Fayet-Iliopoulos term for the hidden $U(1)$ which would introduce a mass splitting]. Otherwise, we shall scan over the remaining parameters to find interesting models.

The two-loop RGEs for the model are given in Appendix A. By taking $m_{S}>m_{H_{+}}$at the high-energy scale, the RGEs naturally drive the soft masses for $m_{H_{+}}^{2}$ to be negative at low energies, triggering hidden symmetry breaking. ${ }^{3}$ The visible sector coupling via the kinetic mixing then determines which field $\left(H_{+}\right.$or $\left.H_{-}\right)$condenses; without loss of generality, we take $\chi$ to be negative, and thus $H_{+}$condenses. Defining $\Delta \equiv \sqrt{\lambda_{S}^{2} \xi-m_{+}^{2} \lambda_{S}^{2} / g_{h}^{2}}$, we have the conditions for a stable minimum with $\left\langle H_{+}\right\rangle=$ $\Delta / \lambda_{S}$ and all other expectation values zero:

$$
\begin{aligned}
& 0 \leq \Delta^{2} \quad 0 \leq m_{-}^{2}+m_{+}^{2}+m_{S}^{2}+2 \Delta^{2} \\
& 0 \leq\left(m_{-}^{2}+m_{+}^{2}+\Delta^{2}\right)\left(m_{S}^{2}+\Delta^{2}\right)-\left|A_{S}\right|^{2} \Delta^{2} .
\end{aligned}
$$

\footnotetext{
${ }^{3}$ We ignore the effect on the running of the kinetic mixing, since such terms always enter suppressed by $\mathcal{O}\left(\chi^{2}\right)$ [20] with an additional loop factor-and are thus equivalent to three-loop order. Of course, it would be interesting to include all of these effects, where then the hidden sector running would then be (extremely weakly) dependent upon the visible sector parameters, and we leave this to future work.
} 
This is reviewed in Appendix B. The hidden gauge boson mass is then given by

$$
m_{\gamma^{\prime}}=\left(\sqrt{2} g_{h} / \lambda_{S}\right) \Delta .
$$

We give two examples of the values obtained scanning over $m_{S}$ and $\alpha_{S} \equiv \frac{\lambda_{S}^{2}}{4 \pi}$ in Fig. 1 .

\section{Symmetry breaking induced by the visible sector}

The mechanism for hidden gauge symmetry breaking promoted in work such as Ref. [20] is via the effective Fayet-Iliopoulos term induced in the hidden sector by the kinetic mixing with the visible Higgs $D$-term. In such a case, the mass-squared's $m_{+}^{2}, m_{-}^{2}$ may be positive provided they are small enough that $\Delta^{2}>0$.

One motivation for this work is that such a case is more difficult to justify in the case of gravity mediation, but it is not implausible, since it can be achieved, for example, through sequestering of the hidden sector. In Sec. IV, we shall examine this case, which is a qualitatively different scenario to that considered in Ref. [20], which considered gauge mediation. In the case of sequestering, we shall assume the gravitino to be much heavier than the hidden sector, but, importantly, that the singlet mass-squared $m_{S}^{2}>0$ and the hidden gaugino mass-squared $M_{\lambda}^{2}$ are of a similar order of magnitude to the hidden Higgs soft terms $m_{+}^{2}, m_{-}^{2}$, while the hidden $A_{S}$ term remains small. This is in contrast to gauge mediation where $m_{S}^{2} \sim M_{\lambda}^{2} \sim 0$.

\section{E. Dark matter candidates}

The model above contains essentially two different dark matter candidates: a Majorana fermion and a Dirac one. ${ }^{4}$ Neglecting the effect of kinetic mixing with the visible neutralino, the fermion mass matrix in the basis $\left(\tilde{\lambda}, \tilde{h}_{+}, \tilde{h}_{-}, \tilde{s}\right)$ corresponding to hidden gaugino, hidden Higgsinos and a hidden singlino is given by

$$
\mathcal{M}_{f}=\left(\begin{array}{cccc}
M_{\lambda} & m_{\gamma^{\prime}} & 0 & 0 \\
m_{\gamma^{\prime}} & 0 & 0 & 0 \\
0 & 0 & 0 & \Delta \\
0 & 0 & \Delta & 0
\end{array}\right)
$$

The Majorana particle is formed from diagonalizing the $\tilde{\lambda}$, $\tilde{h}_{+}$states; in the case of a large $M_{\lambda}$, this leads to a seesaw effect and a low mass. We shall refer to this state as " $\tilde{o}_{1}$," micrOMEGAs notation for the lightest odd particle. Clearly, there will therefore always be a fermion lighter than the hidden gauge boson (to avoid this fate, we would need to add a mass for the hidden singlino). In order for the Dirac fermion formed from $\tilde{h}_{-}, \tilde{s}$ to be the lightest state, we

\footnotetext{
${ }^{4} \mathrm{We}$ are ignoring the possibility of scalar dark matter since, although the model as we have written it contains stable scalars, we expect the symmetries protecting them to be broken at some higher order in the potential allowing them to ultimately decay.
}

would need $\lambda_{S}<\sqrt{2} g_{h}$ and for the Majorana mass $M_{\lambda}$ to be rather small at the high-energy scale (this could happen, for example, in a string model where the modulus corresponding to the gauge coupling does not obtain an $\mathrm{F}$ term), although it is somewhat suppressed in running down to the low scale. Hence, the Dirac fermion scenario is not compatible with radiative-breaking models, but presents an attractive candidate for the visible sector induced breaking. We shall refer to this state as " $\tilde{o}_{7}$." Note that this would not be a good candidate in gauge mediation, as there the singlet scalar would necessarily be lighter than the fermion [20].

In a complete analysis including the couplings and annihilation cross sections, it is necessary to take the mixing with the visible neutralino into account; this we do in Appendix B 2.

Finally, we comment on the (lack of) effect of breaking the residual global symmetry on the above analysis. This could occur via terms in the superpotential or Kähler potential of the form $S^{n}$ suppressed by an appropriate power of a mass scale, such as the string or Planck scale; for example, in string theory, it would be natural to expect terms of the form $S^{n} e^{-a T}$ where $T$ is some modulus charged under a (broken) gauge symmetry from which the residual global symmetry descends-the effect could thus be exponentially suppressed by the expectation value of $T$, and so can, in principle, be naturally arbitrarily small. Since these are small effects, they will not affect the hidden gauge symmetry breaking (the singlet field would obtain a very small expectation value due to the radiative generation of a tadpole term in the potential, which would no longer be prohibited by the symmetry, but of course could be made arbitrarily small), but they will split the Dirac fermion into two Majorana ones with a potentially undetectable mass splitting. However, the lightest of these states, when it is the lightest supersymmetric particle, will be protected from decay by $\mathrm{R}$ parity. This is important when considering the constraints of big bang nucleosynthesis (BBN, and is in contrast to the cases considered in, e.g., Ref. [19]): in principle, any unstable relic with a lifetime greater than $\mathcal{O}(100)$ seconds must obey strict constraints on its density during BBN; see, e.g., Ref. [56]. On the other hand, the model does possess heavy scalars whose decays are protected by this symmetry, and also the heavier component of the Dirac fermion would then decay; however, since the effect can be arbitrarily small, we may simply assume that the lifetimes are many times that of the Universe, and so we can to all intents and purposes treat the symmetry as exact. This is our favored perspective, but we can alternatively make the breaking strong enough that the scalars and heavier components can decay fast enough; for example, a coupling of the form $W \supset \lambda S^{3}$ will induce decays of $S$ with $\Gamma \sim 10^{-2} \lambda^{2} m_{S}$, so $\lambda \geqslant 10^{-11}$ would suffice; similarly, a mass splitting of the fermions of $\Delta m_{\Delta}^{2}$ will allow decays with $\Gamma \sim 10^{-2}\left|g_{h} \chi\right|^{2} m_{\Delta} \frac{\Delta m_{\Delta}^{2}}{m_{\Delta}^{2}}$ which, for 
the values of the couplings considered in this paper, will suffice if $\frac{\Delta m_{\Delta}^{2}}{m_{\Delta}^{2}} \geq 10^{-11}$. We will comment more upon BBN constraints in Sec. III B.

\section{CONSTRAINTS AND DISCOVERY POTENTIAL}

There are already a wealth of constraints on the parameter space of models with dark forces and hidden matter that we must apply in our search over models. However, there are also future experiments which will have the potential to rule further regions out-or make a discovery. In this section, we summarize these current and future constraints and illustrate them by application to a toy model.

\section{A. Limits on the hidden photon}

A summary of various constraints on hidden photons from cosmology (including BBN), astrophysics and laboratory searches for the whole mass and kinetic mixing ranges $10^{-9} \mathrm{GeV} \leq m_{\gamma^{\prime}} \leq 10^{3} \mathrm{GeV}$ and $10^{-15} \leq|\chi| \leq 1$ has been presented for example in Ref. [57] and references therein. For the mass range of interest in this work, the constraints from electroweak precision tests (EWPT) are used as have been presented in Ref. [45], where the strongest constraint is provided by the mass of the $Z$ for most of the parameter space. In the following plots (Figs. 4-6, 8, and 9) of $\chi$ vs $m_{\gamma^{\prime}}$, this is shown as a longdashed approximately horizontal blue line excluding roughly $\chi \geq 3 \times 10^{-2}$. Another constraint comes from the muon anomalous magnetic moment [58] and is dominant for $m_{\gamma^{\prime}}<1 \mathrm{GeV}$ : in the above-mentioned plots of $\chi$ vs $m_{\gamma^{\prime}}$, this is a dashed-dotted brown line at low masses and $\chi>10^{-2}$. There is also a model-dependent constraint from $B A B A R$ searches [45] that might be the most constraining in the region $0.2 \mathrm{GeV} \lesssim m_{\gamma^{\prime}} \lesssim 10 \mathrm{GeV}$, but this only applies if the $\gamma^{\prime}$ cannot decay into hidden sector particles; in the above-mentioned plots of $\chi$ vs $m_{\gamma^{\prime}}$, this is a dashed dark purple line at low masses below $10 \mathrm{GeV}$ and $\chi \sim 2 \times 10^{-3}$. This constraint does apply for most of the supersymmetric models we are considering, where the mass of the $\gamma^{\prime}$ and hidden matter are similar-preventing a decay of $\gamma^{\prime}$ to the hidden sector. However, if the hidden photon can decay to hidden matter, then there is instead a much weaker constraint from the $Z$ width. We require

$$
\frac{\Gamma(Z \rightarrow \text { hidden })}{\Gamma(Z \rightarrow \nu \bar{\nu})} \lesssim 0.008,
$$

which for a single hidden Dirac fermion of mass $M_{X}<M_{Z}$ and unit charge under the hidden $\mathrm{U}(1)$ corresponds to (see also Ref. [59])

$8 c_{W}^{2} s_{W}^{2}\left(\frac{\mathrm{s}_{\phi}}{c_{\epsilon}}\right)^{2}\left(\frac{g_{h}^{2}}{e^{2}}\right)\left(1+2 \frac{M_{X}^{2}}{M_{Z}^{2}}\right) \sqrt{1-4 \frac{M_{X}^{2}}{M_{Z}^{2}}} \lesssim 0.008$,

where $c_{W}, s_{W}$ are the usual cosine and sine of the weak mixing angle respectively; $s_{\phi}$ is defined in Eq. (C7).
For $M_{X} \ll M_{Z}$, this simplifies to $\chi g_{h} \lesssim 0.04$. Clearly, for a small number of hidden particles (and $g_{h}<1$ ), this is a weaker constraint than the measurement of the $Z$ mass.

For $m_{\gamma^{\prime}}$ below $1 \mathrm{GeV}$, there are additional constraints which are shown as grey areas in Fig. 3. The past electron beam dump experiments E141 [60], E137 [61] and E774 [62] have been reanalyzed in Ref. [63] in terms of hidden photons and were found to place limits on small masses $\lesssim 2 m_{\mu}$. In addition, another such limit has been obtained from an electron beam dump experiment at Orsay [64] in Ref. [65]. Recently, two electron fixed target experiments A1 at MAMI in Mainz [66] and APEX at JLab [67] started, which are both searching for hidden photons behind a thin target from bremsstrahlung off an electron beam and which where already able to set first new constraints. Another limit arises in Ref. [68] from the reanalysis of data from a proton beam dump taken at the U70 accelerator at IHEP Serpukhov. At the Frascati DA $\phi$ NE $\phi$ factory, the KLOE2 experiment [69] set further constraints using $e^{+} e^{-}$collisions. However, not only are there limits on the kinetic mixing for very light hidden photons, but excitingly there are also dedicated experiments planned (and partly already running) that can further probe this parameter space with real discovery potential. There are two fixed target experiments (A1 [66] and MESA) in Mainz and three (APEX [67,70], DarkLight [71] and HPS [72]) at JLab. The estimated sensitivities of those experiments are shown in Fig. 3 for the toy model.

\section{B. Constraints from big bang nucleosynthesis}

If a model produces too many high-energy photons, they can dissociate nuclei (such as lithium) and ruin the predictions from nucleosynthesis. The thresholds for these processes are of the order of a few $\mathrm{MeV}$, and so photons produced with energies above this are potentially dangerous. This is typically used to constrain long-lived decaying particles where a photon is among the decay products; due to the rapid interactions of the photons with the plasma, a "zeroth-order" spectrum of energies is produced with a cutoff at $m_{e}^{2} /(22 T)$ (where $m_{e}$ is the electron mass), and so these reactions only activate for temperatures $T$ below $0.01 \mathrm{MeV}$, corresponding to times of the order of $10^{4} \mathrm{~s}$. The strongest constraints are for particles with lifetimes of $10^{8} \mathrm{~s}$. In models with a hidden sector, it is then natural to wonder whether visible photons can be produced, for example, by decays of particles in the hidden sector or the occasional annihilation of the frozen-out dark matter particles.

For a massless hidden photon, hidden sector matter does acquire a small charge under the visible photon (they become "millicharges"), in which case the constraints upon their presence during $\mathrm{BBN}$ are summarized in Ref. [57]. However, since we are considering a massive hidden photon, the diagonalization of the physical states is given in Eq. (C2), from which it can be seen that hidden 
sector states do not couple to the visible photon [cf. also Eq. (C3)]. Moreover, once a hidden photon is produced, the physical state does not oscillate into visible photons ${ }^{5}$ (so the constraints will be very different from, for example, possible sterile neutrinos). It does, however, couple to visible sector matter and decays with a width of $\Gamma \simeq \frac{1}{3} Q^{2} \alpha \chi^{2} c_{W}^{2} m_{\gamma^{\prime}}$ into each light species of charge $Q$, i.e., $\Gamma>10^{-2} \chi^{2} \mathrm{GeV}$, or a lifetime $\tau_{\gamma^{\prime}}<\left(\frac{10^{-11}}{\chi}\right)^{2}\left(\frac{\mathrm{GeV}}{m_{\gamma^{\prime}}}\right) \mathrm{s}$. In this work, we shall be considering $\chi>10^{-5}$, for which the hidden photon will always decay immediately on any cosmological time scales-and so there will be no relic density of hidden photons present.

From the above, we can see that BBN constraints will not affect our dark matter models in much the same way that they do not restrict standard weakly interacting massive particles (WIMPs). However, to be completely strict, let us consider that the annihilation of our dark matter particle will have some nonzero but small branching ratio into visiblesector photons, which we denote $r_{\gamma}$. One could imagine that this would arise from the plasma-induced mixing described above, where $r_{\gamma} \sim \chi c_{W} \frac{m_{P}^{2}}{m_{\gamma^{\prime}}^{2}}$, but given the parameter region we are considering, this will be dominated by loop effects instead. Since the hidden $U(1)$ is not anomalous, the first diagram appears at two loops, yielding $r_{\gamma}<\frac{\alpha^{2}}{(4 \pi)^{2}}<10^{-6}$.

The rate of annihilations of our dark matter candidate $\psi$ into photons per unit volume (assuming that it annihilates entirely through the hidden photon channel) is $\Gamma_{\gamma} / V=$ $r_{\gamma} n_{\psi}^{2}\langle\sigma v\rangle$, where $n_{\psi}$ is the relic density. The strongest bounds for BBN arise for particles of lifetime $10^{8} \mathrm{~s}$ and constrain [56]

$$
m_{\psi} \frac{n_{\psi}}{n_{\gamma}}<5.0 \times 10^{-12} \mathrm{GeV} .
$$

We can therefore take a rough constraint by requiring that our relic particles never produce more photons than such a decaying particle; i.e., $\Gamma_{\gamma} / V<\frac{n_{\gamma}}{m_{\psi}} \times 5.0 \times 10^{-12} \mathrm{GeV} / 10^{8} \mathrm{~s}$ for temperatures lower than $0.01 \mathrm{MeV}$. This yields, roughly,

\footnotetext{
${ }^{5}$ Recall that Eq. (C2) is valid in a vacuum, and during BBN there is a small effect due to the thermal mass for the photon $m_{P}$ in the plasma. Since we must consider temperatures below $0.01 \mathrm{MeV}$, below the electron mass, this is given by $m_{P}^{2} \simeq$ $4 \pi \alpha \frac{n_{e}}{m_{e}} \simeq 4 \pi \alpha \frac{n_{\gamma}}{m_{e}} \eta$, where $n_{e}, n_{\gamma}$ are the densities of electrons and photons, respectively, and $\eta$ is the baryon-to-photon ratio. For $T=0.01 \mathrm{MeV}, \eta=10^{-9}$, this gives an upper bound on the mass of $m_{P} \lesssim 10^{-8} \mathrm{MeV}$. The effect of this additional tiny mass is a minuscule orthogonal rotation of the physical states, whereby the photon and hidden photon mix by an amount $\chi c_{W} \frac{m_{P}^{2}}{m_{\gamma^{\prime}}^{2}}$. If there were a relic population of hidden photons, in principle, a tiny fraction of them could oscillate into visible photons, and we would need to consider their effect on BBNbut, further, for the range of hidden photon masses and kinetic mixing we are considering here, this is clearly completely negligible.
}

$$
r_{\gamma} \lesssim 2 \times 10^{-3}\left(\frac{0.01 \mathrm{MeV}}{T_{c}}\right)^{3}\left(\frac{T_{f}}{50 \mathrm{MeV}}\right),
$$

where $T_{f}$ is the freeze-out temperature (typically $T_{f} \sim m_{\psi} / 20$ ) and we compare the rates at temperature $T_{c}<0.01 \mathrm{MeV}$. This is an overly conservative bound (since the largest disruptive effect of a decaying particle occurs at temperatures much below $0.01 \mathrm{MeV}$ ) but even so is very weak and will not affect the rest of our analysis.

\section{Limits from dark matter}

There are further experimental constraints arising on the dark matter particle, its mass and its interactions. First of all, the dark matter particle should not have a relic abundance in excess of the one measured by WMAP [73],

$$
\Omega_{\mathrm{DM}} h^{2}=0.1123 \pm 0.0035 .
$$

This is a very strict limit and translates to a lower limit on the dark matter (DM) annihilation cross section. We compute the dark matter relic abundance using micrOMEGAs where we have implemented our model. However, while there is an upper limit on the relic abundance, there is no objection to having a dark matter candidate whose abundance is lower than the one measured. In this case, it would then only be a part of the total dark matter (we shall refer to this as subdominant DM), and the remaining dark matter density would consist of other particle(s) such as an axion or axionlike particle whose phenomenology is not the subject of this article-we shall simply assume in such cases that the direct-detection cross sections and interactions with the hidden sector of the additional dark matter are both negligible. In all of our plots, we show parameter points that give an abundance in agreement with the WMAP value in dark green and ones where the DM is subdominant in light green.

Additional constraints apply to the dark matter particle and its scattering cross section on nuclei. It is necessary to distinguish spin-dependent (SD) and spin-independent (SI) scattering. Depending on whether the dark matter particle is a Majorana or Dirac fermion, it has either dominantly SD or SI interactions, respectively. The SI interaction is, moreover, dominated by $\gamma^{\prime}$ exchange, which couples almost exclusively to the proton, particularly at low hidden photon masses (where the mixing can be treated as being effectively between the photon and hidden photon-see Appendix C). The SI interaction is therefore strongly isospin-dependent, and we must rescale limits on the cross sections accordingly (which usually assume equal couplings for protons and neutrons). For the SD interactions, however, the isospin dependence is rather weak, being dominated by $Z$ exchange. Current limits from direct dark matter detection experiments are strongest for SI scattering cross sections $\left(\sim 10^{-42} \mathrm{~cm}^{2}\right)$, while SD cross sections both on protons and on neutrons only start to be excluded at the $10^{-38} \mathrm{~cm}^{2}$ level. 
On the SI side, for the low dark matter masses $(\sim 10 \mathrm{GeV})$ we are interested in, the most relevant constraints come from XENON and CDMS. However, due to the signal claims from DAMA and CoGeNT, ${ }^{6}$ there has been a large debate on the reliability of those constraints, especially at low dark matter masses close to the energy threshold of the experiments. There are also large astrophysical (halo model, dark matter velocity and local dark matter density) and nuclear physics uncertainties that should be taken into account. Even though XENON and CDMS claim to rule out most of the DAMA and CoGeNT preferred regions, the positive signals remain, and there have been various studies of how to reconcile those different results. ${ }^{7}$ We adapt the analysis of Ref. [81] which made a systematic scan taking into account the various uncertainties. There it is found that depending on the halo model, some of the CoGeNT and sometimes even DAMA preferred region is consistent with the exclusions from XENON and CDMS. For the details of the different halo models, see Ref. [81]; we will mostly use their so-called standard model halo (SMH) and in a few cases show the differences that arise when changing for example to a Navarro-Frenk-White (NFW) or an Einasto profile.

We strictly apply the XENON100 and CDMSSi constraints derived in Ref. [81] to the SI scattering cross sections and only show points that are not excluded by any of the two experiments. In the plots of $\sigma_{p}^{\mathrm{SI}}{ }^{8}$ vs $m_{\mathrm{DM}}$ in Sec. IV (see Figs. 7 and 11), the CDMS limit is shown as a dashed turquoise line, while XENON100 is a dasheddotted blue line. For most halo models, CDMS is more constraining at lower masses than XENON100.

In the SD case, there are both for scattering on protons and on neutrons several direct-detection experiments sensitive to the low dark matter masses we are interested in. Different papers also tried to explain the DAMA signal by spin-dependent scattering either exclusively from neutrons [82] or from protons [83]. The former case is, however, not applicable in our models, as the spin-dependent cross sections of the Majorana fermion are always of the same order of magnitude both for protons and neutrons. In the latter analysis, it was shown that for scattering on protons, the DAMA favored region is ruled out by Super-Kamiokande

\footnotetext{
${ }^{6}$ We have not explicitly included the CRESST signal in our search. One of their two signal regions is roughly compatible with both DAMA and CoGeNT signals, although this is still subject to astrophysical uncertainties.

${ }^{7}$ One interesting possibility is to allow isospin-dependent interactions with just the right behavior to suppress the interaction cross section with xenon nuclei [74-80]. We simply note that, although in the case of hidden Dirac fermions the interaction is almost entirely with protons rather than neutrons, in our models this tuning is not possible.

${ }^{8}$ We always show scattering from protons in the plots, hence $\sigma_{p}^{\mathrm{SI}}$, since the constraints are strongest for these, and because our Dirac candidate will couple more strongly to protons than neutrons.
}

(Super-K) due to neutrinos from DM annihilation in the Sun almost independently of the annihilation channel. Additionally, the cross sections required in both scenarios are more than one order of magnitude above the largest ones that can be obtained in our models. Therefore, if the explanation of the DAMA (and CoGeNT) signals is confirmed as arising from spin-dependent scattering, it would rule out the models considered in this paper. Hence, we do not study this in more detail and simply apply the various spin-dependent scattering direct-detection constraints. Until June 2011, PICASSO for the lightest and COUPP for the slightly larger masses were the most constraining experiments for SD scattering on protons [84]. Very recently, a new direct-detection experiment SIMPLE [85] has published a limit on the SD scattering cross section on protons which in the low mass range is one order of magnitude stronger than previous experiments (for a critique of their limit, see Ref. [86] and the collaboration's response [87]). There is also a quite strong limit from Super-K using neutrino fluxes produced by dark matter annihilation in the Sun which, however, only applies to dark matter masses above $20 \mathrm{GeV}$ (only neutrino-induced upward through-going muons have been used in this analysis which leads to a quite high-energy threshold and therefore a sensitivity only to larger DM masses) [88]. ${ }^{9}$ For SD scattering on neutrons, there are limits from XENON10 [90], Zeplin [91] and CDMS [16,92,93], the strongest of which, set by XENON10 for the mass range of interest in this paper, is less constraining than the SIMPLE limit.

In the following analysis, we use all constraints from SD scattering both on protons and on neutrons with the exception of SIMPLE as strict exclusions and show only points consistent with those limits. As there has been criticism of SIMPLE's limit, we will not apply this universally but rather show how our results change when taking it into account. In the plots of $\sigma_{p}^{\mathrm{SD}}$ vs $m_{\mathrm{DM}}$ in Sec. IV (see Figs. 7 and 10), the exclusion lines for the different experiments are as follows: SIMPLE short-dashed brown line, Super-K dashed black line, PICASSO long-dashed orange line, COUPP2011 dashed-dotted turquoise line, COUPP2007 dotted blue line and KIMS long-dashed green line. The plots of $\sigma_{n}^{\mathrm{SD}}$ vs $m_{\mathrm{DM}}$ in the same figures show the limits of XENON10 as dashed-dotted blue, Zeplin as dotted pink and CDMS as dashed turquoise lines.

Those constraints on the scattering cross section can strictly only be applied to particles that actually constitute the entire dark matter density. If the dark matter is subdominant, however, the limits on its scattering cross section have to be rescaled accordingly: the local density $\rho_{\psi}$ of a dark matter candidate $\psi$ relates to the local total DM density $\rho_{\mathrm{DM}}$ as their abundances

\footnotetext{
${ }^{9}$ There is another more recent analysis [83] with limits for smaller masses. Application of these limits taking into account the annihilation details and branching ratios is beyond the scope of this work and left for future works [89].
} 


$$
\frac{\rho_{\psi}}{\rho_{\mathrm{DM}}}=\frac{\Omega_{\psi}}{\Omega_{\mathrm{DM}}}
$$

and so do the limits that are set by direct-detection (DD) experiments. Thus, an experimental bound on $\sigma_{\mathrm{DD}}$ translates into an actual bound on the scattering cross section $\sigma_{\psi}$ of $\psi$ as

$$
\sigma_{\psi}=\sigma_{\mathrm{DD}} \frac{\Omega_{\psi}}{\Omega_{\mathrm{DM}}} .
$$

This means that direct-detection constraints on the scattering cross section become less potent for subdominant DM particles. ${ }^{10}$

\section{Application to toy model}

To illustrate the above constraints/future experimental reach, and more importantly provide a comparison to the more complete model of Sec. II that we shall investigate in Sec. IV, here we shall consider a toy model. This is the simplest possible dark sector: a Dirac fermion $\psi$ with unit charge only under the (massive) hidden U(1). We shall not include any Higgs sector - the U(1) could, after all, naturally have a GeV scale mass via the Stückelberg mechanism $[25,29]$ - so we will not consider how the dark matter particle becomes massive. This is essentially the model considered in Refs. [46-50] except that we shall insist on the relation (2.4); the parameters are the dark matter mass $m_{\psi}$, hidden photon mass $m_{\gamma^{\prime}}$, kinetic mixing $\chi$ and the tuning parameter $\kappa$.

\section{Constraints and future searches}

The DM can annihilate through and/or into hidden photons according to the diagrams shown in Fig. 2. Whereas the left diagram is possible for all DM masses, the right one is kinematically only accessible when $m_{\psi} \geq m_{\gamma^{\prime}}$. The left diagram also leads to a resonant enhancement of the annihilation cross section and accordingly to a dip in the relic abundance for $m_{\gamma^{\prime}}=2 m_{\psi}$. This can been seen in Fig. 3 where we show the relic abundance for a dark matter mass of $6 \mathrm{GeV}$ (left plot) and $7 \mathrm{GeV}$ (right) as a function of the kinetic mixing $\chi$ and the hidden photon mass $m_{\gamma^{\prime}}$. The grey areas are excluded by beam dump experiments (curves on the left side of the plot), muon and electron anomalous magnetic moment (top left corner of the plot) as well as the $B A B A R$ search and EWPT (curves at large $\chi$ and $m_{\gamma^{\prime}}$ ) as described in Sec. III A. The thin dark green band is the region which gives the correct WMAP abundance (3.5), while in the light green areas, the DM candidate is subdominant. The white region is excluded since it gives a too large relic abundance. For very small hidden photon masses, annihilation proceeds only via the left diagram of Fig. 2 and is essentially independent of $m_{\gamma^{\prime}}$. Therefore, the relic abundance is given by the kinetic mixing only, which itself is determined by the

\footnotetext{
${ }^{10}$ This is obviously based on the reasonable assumption that the local DM has the same content of different DM contributions as averaged over the whole Universe.
}

hidden gauge coupling up to a factor $\kappa .{ }^{11}$ The colored lines with named labels represent the future searches mentioned in Sec. III A, which as can be seen from the plot will probe portions of the interesting parameter space.

The DM particle considered for the toy model in this section can also scatter elastically on nuclei. As it is a Dirac fermion, this process is spin-independent, and the corresponding cross sections can be compared to the positive observations of DAMA and CoGeNT. The results in Fig. 3 are given for the SMH (left plot) and Einasto (right plot). In the former case, a part of the CoGeNT allowed regionand in the latter case, both of the CoGeNT and DAMA allowed regions - are not excluded by the other experiments (CDMS and XENON100). The band in purple/red corresponds to the $90 \%$ (lighter) and 99\% (darker) contours where the correct cross section for CoGeNT/DAMA can be obtained, respectively. The blue band on the right plot gives the region where both DAMA and CoGeNT can be explained at the same time. At the place where these bands overlap with the dark green region, the DM candidate that fits the respective DD experiment is also providing all of the dark matter in the Universe. In the larger part, however, where the colored bands are on top of the light green area, the DM particle explains the corresponding DD signal while only contributing subdominantly to the total DM.

Constraints on SI scattering from CDMS and XENON100 do not apply to the low DM mass of $6 \mathrm{GeV}$ used in the left plot of Fig. 3. In the right plot, however, for a DM mass of $7 \mathrm{GeV}$ in the Einasto profile, the scattering cross section is constrained by XENON100 (below the reach of CDMS) which is shown as a blue line excluding all the parameter space above it (where there are dashed vertical lines). Where the XENON100 exclusion bound enters the WMAP allowed (light green) region, the limit is rescaled as described above to correspond to the appropriate dark matter density. However, outside of this region, it is not rescaled-the straight line shown corresponds to the behavior for a constant dark matter density equal to that observed. This accounts for the sudden change in gradient. Note that relation (2.4) has a significant effect upon the behavior of this bound. Outside of the WMAP allowed region, i.e., when we are applying the XENON100 bound for a fixed dark matter density, the corresponding cross section follows a contour of $\chi \propto m_{\gamma^{\prime}}$, rather than $\chi \propto m_{\gamma^{\prime}}^{2}$ which we would find if we were instead keeping $g_{h}$ constant. This arises since the cross section behaves as

$$
\sigma_{\mathrm{DD}} \propto \frac{\chi^{2} g_{h}^{2}}{m_{\gamma^{\prime}}^{4}} \propto \frac{\chi^{4}}{m_{\gamma^{\prime}}^{4}} .
$$

This explains the straight line portion of the XENON100 bound in the log- $\log$ plots of Fig. 3, where $\kappa$ is held fixed. Note how this changes when we take rescaling into account: since the thermal-averaged $\psi-\bar{\psi}$ annihilation cross section

\footnotetext{
${ }^{11}$ We have investigated varying $\kappa$ within the an order of magnitude, and it does not make a qualitative difference to the plots.
} 

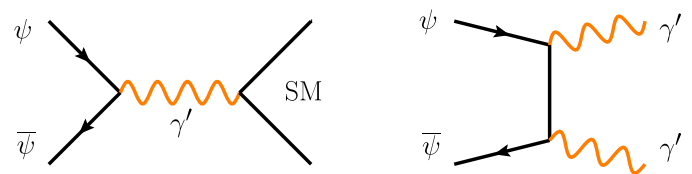

FIG. 2 (color online). Annihilation diagrams: $s$-channel annihilation on the left, resonant at $m_{\gamma^{\prime}}=2 m_{\psi}$; $t$-channel on the right, accessible and dominant when $m_{\psi}>m_{\gamma^{\prime}}$.
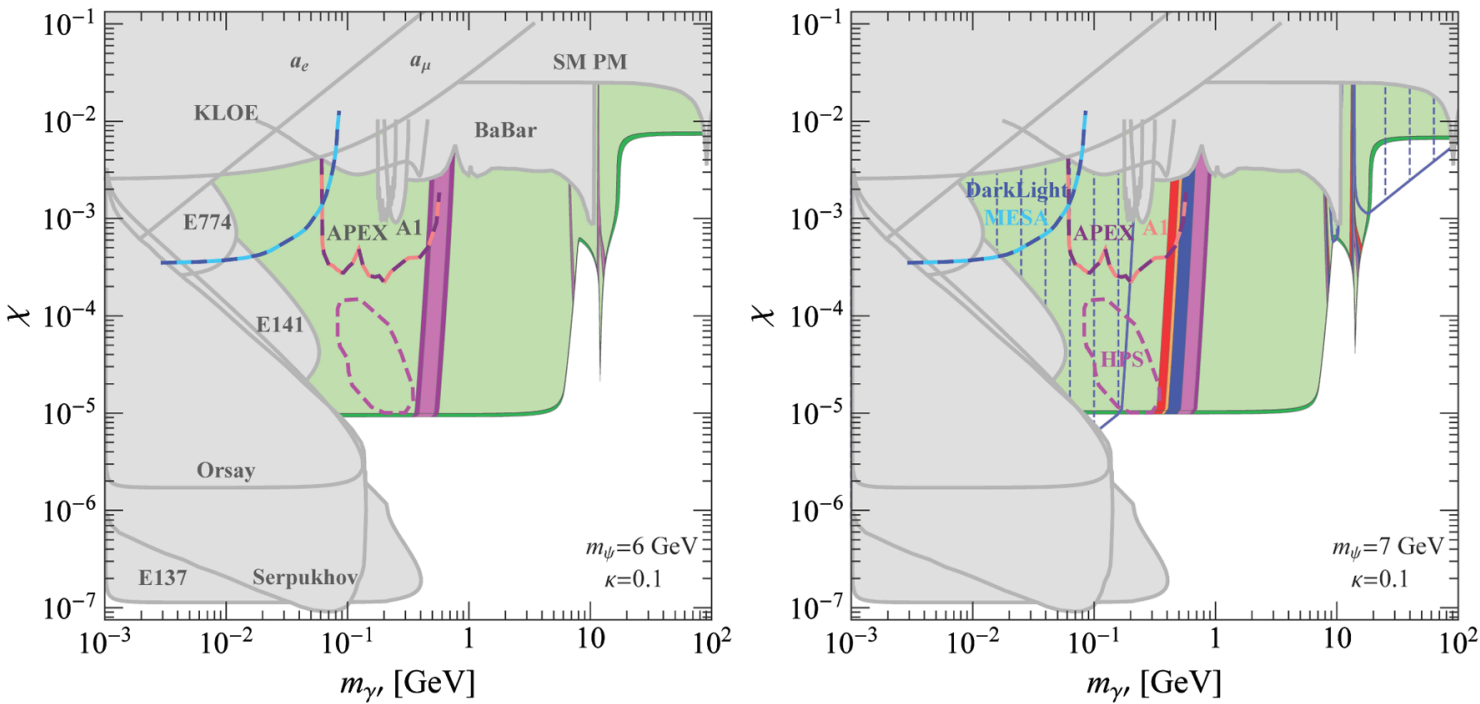

FIG. 3 (color online). DM relic abundance and direct-detection cross section in agreement with CoGeNT and DAMA for a Dirac fermion DM candidate with a mass of $6 \mathrm{GeV}$ in the SMH (left) and $7 \mathrm{GeV}$ in the Einasto halo model (right) with $\kappa=0.1$ for both. The grey labeled regions are excluded by different searches as shown in the left plot: the anomalous magnetic moment of electron and muon $a_{e}$ and $a_{\mu}$, EWPT, model dependent BABAR searches, $e^{+} e^{-}$collisions in KLOE, the electron fixed target experiments A1 and APEX, the electron beam dump experiments E774, E141, E173, and Orsay as well as the proton beam dump at Serpukhov (cf. Sec. III A for details and references). The colored lines with corresponding labels in the right plot correspond to sensitivities of the already running experiments A1 and APEX as well as the planned fixed target experiment HPS (see Sec. III A). The thin dark green regions (horizontal line at $\chi \sim 10^{-5}$ continuing towards the resonance) give the correct relic abundance, light green (grey area above $\chi \sim 10^{-5}$ ) is subdominant DM, and white is overabundant and therefore excluded. The scattering cross sections are such that they can explain the CoGeNT observation in the purple area, the DAMA observation in the red area, and both experiments at the same time in the blue area which is only possible for the Einasto profile in the right plot (direct detection regions as almost vertical dark grey bands). The blue line in the right hand plot is the XENON100 bound which excludes all the dashed shaded area above the line.

multiplied by speed $\left\langle\sigma_{\text {Ann }} v\right\rangle$ for fixed dark matter and hidden photon mass is proportional to either $g_{h}^{2} \chi^{2}$ or $g_{h}^{4}$, which according to Eq. (2.4) translates into $\left\langle\sigma_{\mathrm{Ann}} v\right\rangle \propto \chi^{4}$, and as the relic density is proportional to $1 /\left\langle\sigma_{\mathrm{Ann}} v\right\rangle$, we find

$$
\sigma_{\psi} \propto \frac{\chi^{4}}{m_{\gamma^{\prime}}^{4}} \frac{1}{\left\langle\sigma_{\mathrm{Ann}} v\right\rangle} \propto \frac{1}{m_{\gamma^{\prime}}^{4}} .
$$

Hence, the rescaled XENON100 exclusion bound is approximately a vertical line on the plot, as can be seen in Fig. 3 where the blue line meets the green band.

\section{Example data point}

To illustrate the above model, let us consider an example set of values that satisfies all of the constraints and explains the signals while constituting all of the dark matter. Taking, as in Fig. 3, $\kappa=0.1$ and a hidden dark matter particle of $6 \mathrm{GeV}$, we find $\chi=1.2 \times 10^{-5}$, giving via Eq. (2.4) a hidden gauge coupling of $g_{h}=0.053$; and masses for the hidden photon between 0.26 and $0.33 \mathrm{GeV}$ with width between $2.5 \times 10^{-13}$ and $3.5 \times 10^{-13} \mathrm{GeV}$ for the given mass range. This yields the dark matter density within three standard deviations of the WMAP7 result (3.5), while the rescaled direct-detection nucleon cross sections range from $2.7 \times$ $10^{-40} \mathrm{~cm}^{2}$ for the smaller hidden photon mass and $1.1 \times$ $10^{-40} \mathrm{~cm}^{2}$ for the higher, which can explain the CoGeNT signal with the standard halo model, or both DAMA and CoGeNT when Einasto is used [81] — the interaction is almost entirely spin-independent and with the proton. Since the dark matter is so much heavier than the hidden photon here, it annihilates almost entirely via the $t$-channel diagram of Fig. 2 which is unsuppressed by the kinetic mixing relative to the first; this is then almost independent of the hidden photon mass; hence, the contour in Fig. 3 of dark matter density matching the observed one is approximately horizontal up to masses near that of the dark matter particle. 

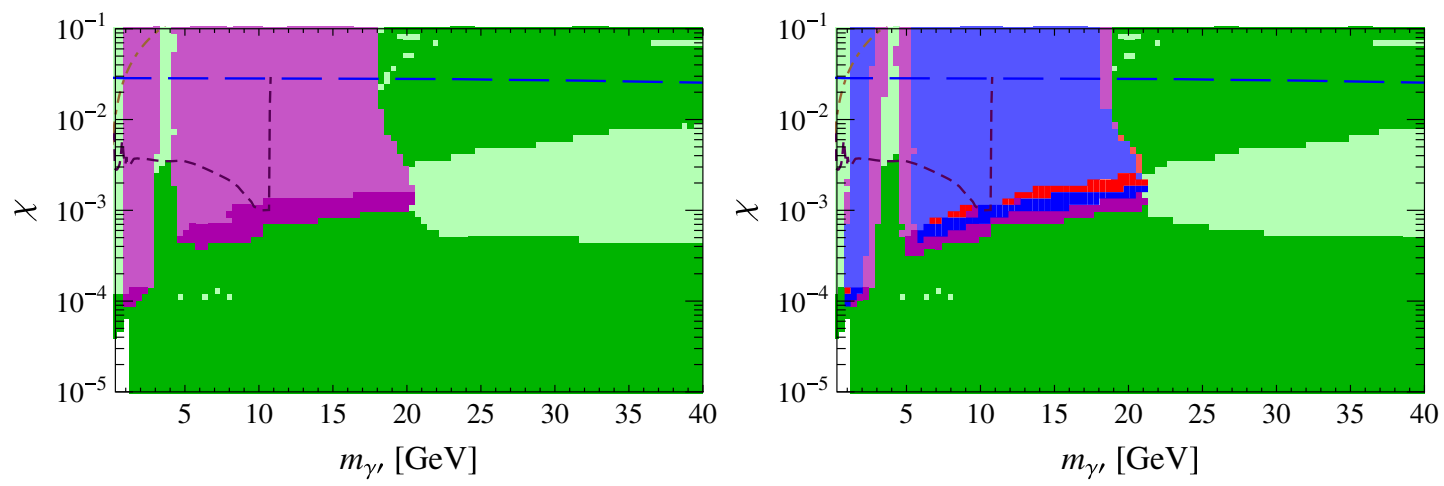

FIG. 4 (color online). Scan over hidden photon mass $m_{\gamma^{\prime}}$, kinetic mixing $\chi$ and hidden Dirac fermion mass $m_{\psi}$ over the range from 0.8 to $25 \mathrm{GeV}$, using (left) the SMH and (right) Einasto constraints given in Ref. [81]. Dark colored regions indicate that the correct relic abundance can be found, and lighter colors indicate that the hidden fermion is a subdominant dark matter candidate. Green regions (lightest and second lightest grey) are thus simply WMAP allowed, but also shown are regions where the direct-detection cross section can explain the signal from either CoGeNT (purple), DAMA (red) or both at the same time (blue or darker shades of grey). The parameter $\kappa$ has been fixed to 1 . For subdominant DM, the scattering cross sections have been rescaled. All points shown in the figure are in agreement with all direct-detection constraints. The constraint from electroweak precision tests is shown as the almost horizontal long-dashed blue line, the (model-dependent) $B A B A R$ limit is shown as a dashed dark line, and the muon $g-2$ constraint is given as dashed-dotted line at the top left corner of the plot.

\section{Scanning over dark matter masses}

To fully examine the parameter space of these models, we performed a scan over the mass $m_{\psi}$ of the particle $\psi$ for values between 0.8 and $25 \mathrm{GeV}$ while also varying the kinetic mixing and hidden photon mass. The resulting scatter plots are shown in Fig. 4 for two different halo models (for details of the halo models, see Ref. [81]). For these plots, the parameter $\kappa$ was fixed to its central value of one. The coloring is as follows: dark shades correspond to the dark matter candidate producing the observed relic abundance, and lighter shades indicate it is subdominant; green regions do not correspond to an experimental signal but are not excluded; purple corresponds to explaining the CoGeNT signal; the DAMA signal is explained in the red regions; CoGeNT and DAMA signals are explained simultaneously in the blue regions. For the SMH, the CoGeNT and DAMA signal regions do not overlap, and this is reflected in the absence of blue in our SMH plots. However, for the Einasto (Fig. 4, right) halo model, there is a small region of overlap in mass - cross-section space for the signal regions, which translates into blue regions of our plots with that choice of halo (Figs. 4, right, and 9, right-we have also checked that the situation is very similar for the NFW halo model). However, in the following section, we shall use mostly the standard halo model; the choice of halo has a more dramatic effect on the presence (or otherwise) of overlap of the signal region than the allowed parameter space of our models.

In the previous subsection, we found that models with very low mass hidden photons $(<\mathrm{GeV})$ coupled to a Dirac fermion of mass of a few $\mathrm{GeV}$ can be consistent with all constraints, can form the entirety of the dark matter and can explain the direct-detection signals. As can be seen from the right plot in Fig. 4, this is also possible for a thin (red) band of the parameter space at higher masses. Since the direct-detection signals are only explained for a dark matter particle in a narrow range of masses between 5.5 and $8.9 \mathrm{GeV}$, we see that this band begins at hidden photon masses equal to the dark matter mass (indeed this data is almost enough to read off the parameters of the models from the scatter plot). This means that the dark matter annihilation only proceeds via the $s$-channel exchange of Fig. 2; for hidden photons lighter than this, the $t$-channel annihilation is resonant, explaining the polelike shape of the purple patch. A sample model constituting the entirety of the dark matter, obeying all constraints and explaining DAMA and CoGeNT (when Einasto is used) with $m_{\psi}=6 \mathrm{GeV}$ and a spin-independent nucleon cross section of $1.1 \times 10^{-40} \mathrm{~cm}^{2}$, has $\kappa=1, \chi=$ 0.0016 (thus, $g_{h}=0.72$ ) and $m_{\gamma^{\prime}}=14.1 \mathrm{GeV}$. The hidden photon is then quite wide; it has width $0.14 \mathrm{GeV}$, almost entirely decaying into the dark matter.

\section{ANALYSIS OF A SUPERSYMMETRIC DARK SECTOR}

In this section, we describe the results of a scan over the parameter space of the model of Sec. II, constrained by dark matter abundance and direct-detection cross sections. This was achieved by implementation of our models in micrOMEGAs [51-55] which automatically computes all of the required annihilation cross sections and integrates the Boltzmann equations to give the relic density. It also calculates the direct-detection cross sections for protons and nucleons. Generation of the model files was performed using LanHEP [94-98]. We included all of the interactions between the hidden and visible sector including the neutralino mixing (described in Appendix B 2) and Higgs portal term (described in Appendix E) which we believe to be novel results; as a result, there is some dependence on the visible sector spectrum and couplings. Since we were investigating the effects of gravity mediation, and for minimality, we chose the visible 

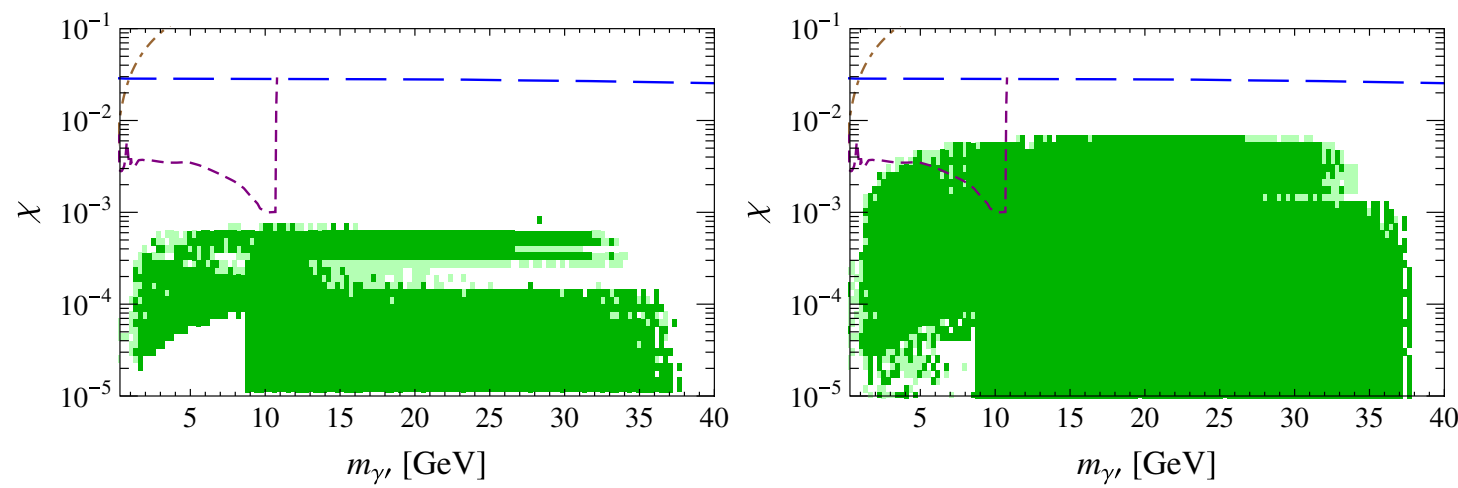

FIG. 5 (color online). Allowed space of models with radiatively induced breaking, showing hidden photon mass against the magnitude of kinetic mixing. Dark green areas (darker grey) allow for the correct dark matter relic density and light green (lighter grey) for subdominant dark matter. The lines represent the constraints from EWPT (long-dashed blue), model-dependent $B A B A R$ search (dashed dark line) and muon $g-2$ (dashed-dotted). The SMH has been used, and all DD constraints are imposed except for the SIMPLE exclusion limit. Left: $\kappa=1$, right: $0.1 \leq \kappa \leq 10$.

sector to consist of the MSSM with a Higgs mass above the LEP bound and the lightest visible sector neutralino in the range 100 to $200 \mathrm{GeV}$; the effect of changing the spectrum within these ranges leads to quantitative changes of a few percent, but not qualitative ones.

As mentioned in Sec. II, we shall take the kinetic mixing parameter $\chi<0$ so that the field $H_{+}$obtains a vacuum expectation value rather than $H_{-}$. Due to the symmetry of the model, this is entirely a matter of choice, so the physical results are unchanged by changing the sign. Therefore, and for ease of comparison with the previous section, in our plots of Figs. 3-6, 8, and 9, we show the magnitude of $\chi$.

\section{A. Radiative breaking domination}

\section{Parameter scan}

Here we perform a scan over $\lambda_{S}, \chi$ and $m_{\gamma^{\prime}}$ in order to find parameter combinations which give a light dark matter candidate (mass in the range between 0.8 and $20 \mathrm{GeV}$ ) which, as mentioned in Sec. IIE, we find to be exclusively a Majorana fermion $\tilde{o}_{1}$. We insist that $\lambda_{S}$ and the hidden gauge coupling inferred from $\chi$ remain perturbative; this places an upper limit upon $\chi$ via Eq. (2.4). We are interested in light hidden gauge bosons, so we choose a maximum value of $m_{\gamma^{\prime}}$ of $40 \mathrm{GeV}$. The low-energy parameters are found by choosing boundary conditions at the high-energy scale $\left(10^{16} \mathrm{GeV}\right)$ and running down; this ensures that we have bona fide consistent models at the low-energy scale, rather than choosing the parameters completely ad hoc. This search uses the RGE engine from SoftSUSY [99].

We then input the results of the scan into micrOMEGAs to obtain the corresponding relic abundance and scattering cross sections. Results for kinetic mixing against hidden photon mass are shown in Figs. 5 and 6 for $\kappa=1$ and for a scan over $\kappa$ in the range 0.1 to 10 . Depending on whether the relic abundance corresponds to the total DM abundance, the points are shown in dark green or in light green if it is subdominant. Clearly, allowing for variation in $\kappa$ has a large effect on the allowed space of models, in stark contrast to the toy model in Sec. III D where the parameter space could be filled without varying $\kappa$.

The spin-dependent and spin-independent direct-detection cross sections are shown in Fig. 7, where $\kappa$ has been scanned over and we again use the rescaling procedure for subdominant dark matter. The dark matter particle considered in this subsection $\tilde{o}_{1}$ is a Majorana fermion and therefore has greatly suppressed spin-independent scattering on nuclei (so there is little chance of explaining the DAMA or CoGeNT signals via spin-independent scattering with such a model); ${ }^{12}$ however, the obtained spin-dependent cross sections are quite large, and some are even already excluded by current experiments (the experiments were mentioned in Sec. IIIC). The most stringent constraint arises from the SIMPLE experiment for SD scattering on protons which cuts out many parameter points for dark matter masses above $\sim 6 \mathrm{GeV}$; the effect of this is illustrated by showing the parameter scan before SIMPLE is included in Fig. 5 and afterward in Fig. 6. Since the spin-dependent and spin-independent cross sections are related, the SIMPLE limit removes a large portion of the parameter space with larger values of spin-independent scattering direct-detection cross sections. This is illustrated in Fig. 7, which presents the different scattering cross sections. The top plot contains all the spin-dependent cross sections on protons with the experimental bounds. At the bottom, the corresponding spin-independent cross sections are shown on the left and the spin-dependent ones on neutrons on the right. In those two plots, yellow and orange points indicate models that lie in the top plot above the SIMPLE exclusion contour for spin-dependent scattering on protons (points in yellow have a subdominant and points in orange the total DM

\footnotetext{
${ }^{12}$ Here, we do not study different halo profiles as they only effect the potential signals in direct-detection experiments for which the required cross sections are several orders of magnitude above those obtained in the models of Sec. IVA. Therefore, the results presented for the SMH do not differ for other halo models.
} 

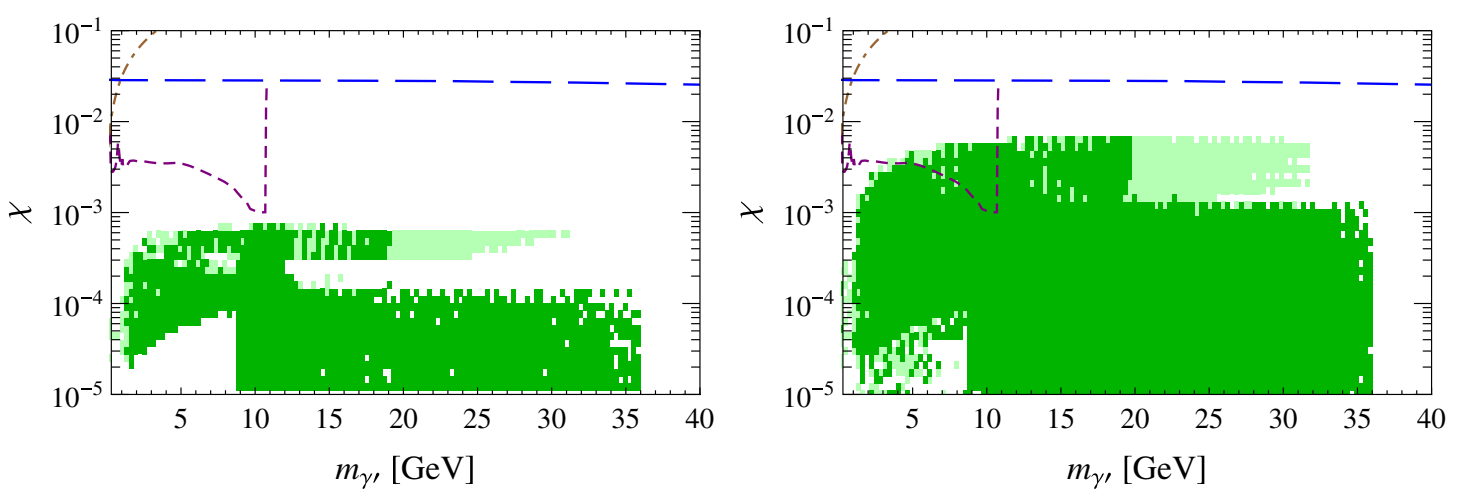

FIG. 6 (color online). Allowed space of models with radiatively induced breaking, showing hidden photon mass against the magnitude of kinetic mixing. Dark green areas (darker grey) allow for the correct dark matter relic density and light green (lighter grey) for subdominant dark matter. The lines represent the constraints from EWPT (long-dashed blue), model-dependent $B A B A R$ search (dashed dark line) and muon $g-2$ (dashed-dotted). The SMH has been used, and all DD constraints are imposed including the SIMPLE exclusion limit. Left: $\kappa=1$, right: $0.1 \leq \kappa \leq 10$
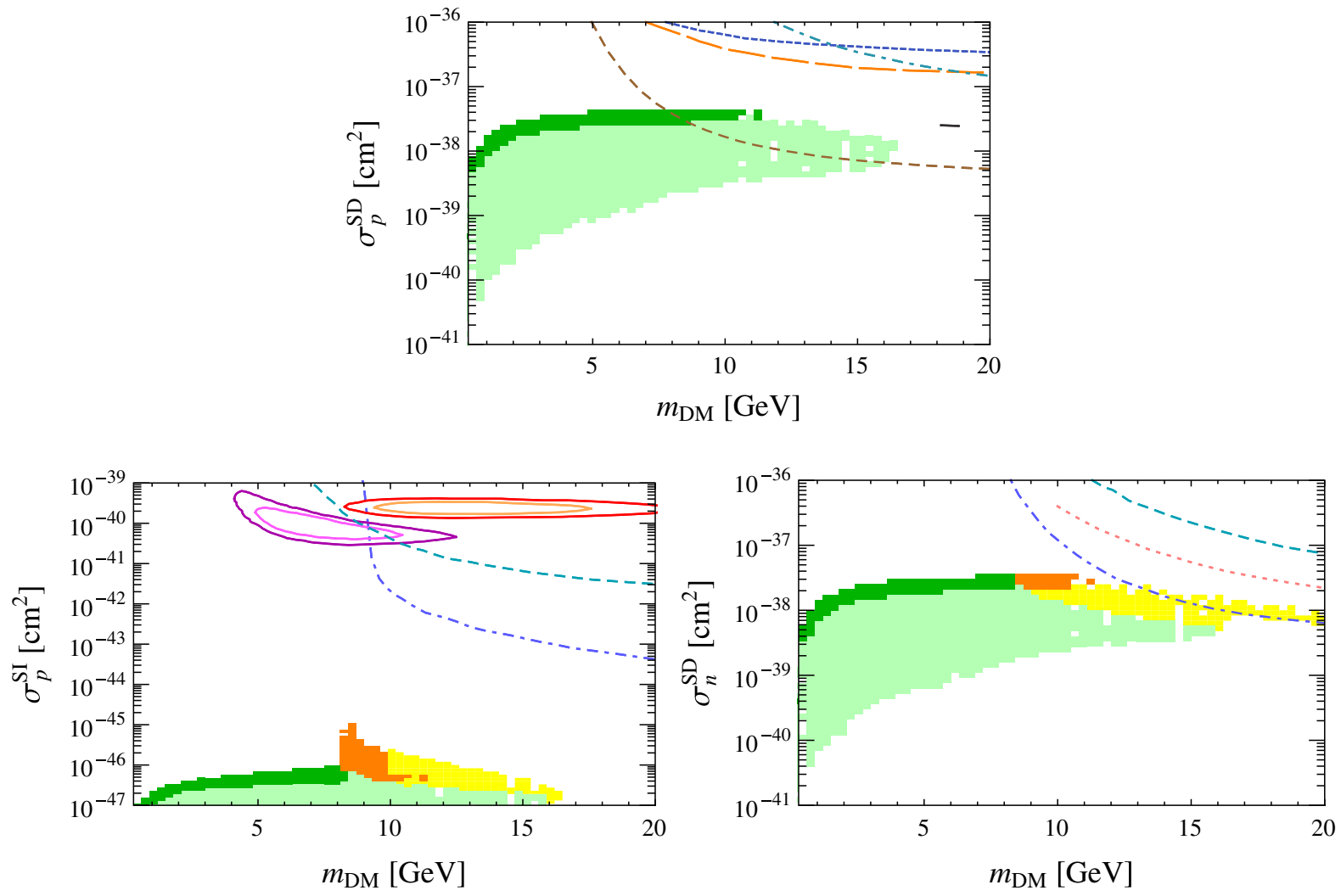

FIG. 7 (color online). Direct detection cross sections for radiatively induced breaking where the DM candidate is a Majorana fermion, using SMH, scanning over $0.1 \leq \kappa \leq 10$. Dark green areas (darker grey) allow for the correct dark matter relic density and light green (lighter grey) for subdominant dark matter. Top: spin-dependent scattering cross section $\left(\sigma_{p}^{\mathrm{SD}}\right)$ on protons with experimental exclusion contours; SIMPLE is shown as the lowest-lying, short-dashed brown curve, and above it is the PICASSO long-dashed orange line, there above the COUPP2011 dashed-dotted turquoise limit, then the COUPP2007 dotted blue line and at the right of the plot starts the dashed black Super-K limit. Left bottom: spin-independent scattering cross section on protons $\left(\sigma_{p}^{\mathrm{SI}}\right)$ together with signal contours from CoGeNT (purple ellipsoid more to the left) and DAMA (red ellipsoid more to the right) as well as exclusion limits from CDMS (dashed turquoise) and XENON100 (dashed-dotted blue). Right bottom: spin-dependent scattering cross section on neutrons $\left(\sigma_{n}^{\mathrm{SD}}\right)$ together with limits from XENON10 (dashed-dotted blue), Zeplin (dotted pink) and CDMS (dashed turquoise). In both plots on the bottom, points in yellow and orange lie above the SIMPLE limit while giving a subdominant and total DM abundance, respectively. 
abundance). As can be seen from the right bottom plot, the SIMPLE limit for spin-dependent scattering on protons is more constraining than limits from scattering on neutrons where XENON10 can exclude only few models.

As mentioned above, the fact that the hidden sector dark matter candidate is a Majorana fermion leads to extremely small spin-independent scattering cross sections. They do, however, obtain a contribution from the Higgs portal term, which in supersymmetric theories is always present. We describe this in detail in Appendix E, where we also derive a simple approximation for the contribution of the Higgs portal term which agrees well with the results seen in Fig. 7:

$\sigma_{N}^{\text {SI,portal }} \sim 10^{-45} \mathrm{~cm}^{2} \times\left(\frac{m_{\tilde{o}_{1}}}{m_{N}+m_{\tilde{o}_{1}}}\right)^{2}\left(\frac{\chi s_{W}}{0.001}\right)^{2}\left(\frac{\mathrm{GeV}}{m_{\gamma^{\prime}}}\right)^{2}$.

There is also a somewhat smaller and more spectrumdependent contribution from squark exchange. For the Majorana fermion DM of this section, the spin-independent nuclear cross sections are very similar for scattering on protons and on neutrons; hence, we have written $\sigma_{N}^{\text {SI,portal }}$ with "N" to denote nucleons; in the plots (Fig. 7), the cross sections on protons $\left(\sigma_{p}^{\text {SI,portal }}\right)$ are shown, which also allows direct comparison with the next subsection.

Our results in this subsection are largely independent of the halo model applied to the spin-independent scattering limits, as the corresponding cross sections are much below the experimental reach.

\section{Example model}

To better understand the types of models that we find, since the plots can only show two-dimensional parameter spaces, here we give an example of one of the models that satisfies all experimental constraints and provides the entire dark matter density. We take $\kappa$ to be unity and the soft masses $m_{H_{ \pm}}$ approximately $100 \mathrm{GeV}$ at the high-energy scale. We then run the parameters down and adjust at the high scale to find appropriate values at low energies; thus, $m_{S}$ is somewhat larger and drives the soft hidden Higgs masses to become tachyonic. The parameters at low $(10 \mathrm{GeV})$ and high $\left(10^{16} \mathrm{GeV}\right)$ energy scales are given in Table I along with the spectrum at low energies after hidden gauge symmetry breaking. The dark matter candidate is then the Majorana fermion $\tilde{o}_{1}$, having a mass of $5.2 \mathrm{GeV}$ and yielding a density of
TABLE I. Hidden sector parameters and particle masses for an example gravity-mediated model, yielding the entire dark matter density $\Omega_{\tilde{o}_{1}} h^{2}=0.112$. The direct-detection nucleon cross sections are $\sigma_{p}^{\mathrm{SI}}=3.6 \times 10^{-47} \mathrm{~cm}^{2}$ and $\sigma_{p}^{\mathrm{SD}}=2.5 \times 10^{-38} \mathrm{~cm}^{2}$, well outside the reach of current experiments.

\begin{tabular}{|c|c|}
\hline \multicolumn{2}{|c|}{ High scale parameters } \\
\hline$\kappa$ & -1.0 \\
\hline$\chi$ & -0.0008 \\
\hline$\alpha_{h}$ & 0.0031 \\
\hline$\alpha_{S}$ & 0.011 \\
\hline$M_{\lambda}$ & $21.4 \mathrm{GeV}$ \\
\hline$m_{H_{+}}^{2}$ & $(101)^{2} \mathrm{GeV}^{2}$ \\
\hline$m_{H_{-}}^{2}$ & $(101)^{2} \mathrm{GeV}^{2}$ \\
\hline$m_{S}^{2}$ & $(418)^{2} \mathrm{GeV}^{2}$ \\
\hline$A_{S}$ & $-0.2 \mathrm{GeV}$ \\
\hline \multicolumn{2}{|c|}{ Low scale parameters } \\
\hline$\kappa$ & -1.0 \\
\hline$\chi$ & -0.0005 \\
\hline$\alpha_{h}$ & 0.003 \\
\hline$\alpha_{S}$ & 0.010 \\
\hline$M_{\lambda}$ & $20.7 \mathrm{GeV}$ \\
\hline$m_{H_{+}}^{2}$ & $-66.8 \mathrm{GeV}^{2}$ \\
\hline$m_{H_{-}}^{2}$ & $-68.9 \mathrm{GeV}^{2}$ \\
\hline$m_{S}^{2}$ & $(406)^{2} \mathrm{GeV}^{2}$ \\
\hline$A_{S}$ & $-1.5 \mathrm{GeV}$ \\
\hline Particle & Mass $(\mathrm{GeV})$ \\
\hline$\tilde{o}_{7}$ & 14.0 \\
\hline$\tilde{o}_{1}$ & 5.2 \\
\hline$\tilde{o}_{2}$ & 25.9 \\
\hline$\gamma^{\prime}$ & 11.6 \\
\hline$H_{+}$ & 11.6 \\
\hline$H_{-}, S$ & $7.7,406$ \\
\hline
\end{tabular}

$\Omega_{\tilde{o}_{1}} h^{2}=0.112$. The spin-independent nuclear direct detection cross section is $\sigma_{p}^{\mathrm{SI}}=3.6 \times 10^{-47} \mathrm{~cm}^{2}$, the spindependent cross section being $\sigma_{p}^{\mathrm{SD}}=2.5 \times 10^{-38} \mathrm{~cm}^{2}$. The mass of the hidden photon and hidden Higgs is $11.6 \mathrm{GeV}$, and they have widths of $6.7 \times 10^{-8} \mathrm{GeV}$ and $4.8 \times 10^{-8} \mathrm{GeV}$, respectively, the latter decaying mostly to charm and $b$ quarks.

For the SPS1b data point [100], the full neutralino mass matrix in the basis $\left(B_{0}, W_{0}, h_{u}^{0}, h_{d}^{0}, \tilde{\lambda}, \tilde{h}_{+}\right)$is (in $\mathrm{GeV}$ )

$$
\mathcal{M}_{\text {neutralino }}=\left(\begin{array}{cccccc}
166 & 0 & -2.73 & 43.8 & -0.01 & -0.01 \\
0 & 310 & 2.73 & -79.9 & 0 & 0 \\
-2.73 & 2.73 & 0.00 & -511 & 0 & 0 \\
43.8 & -79.9 & -511 & 0 & 0 & 0 \\
-0.01 & 0 & 0 & 0 & 20.7 & 11.6 \\
-0.01 & 0 & 0 & 0 & 11.6 & 0
\end{array}\right)
$$

with eigenmasses 5.2, 25.9, 164, 298, 516, and $530 \mathrm{GeV}$. For the same data point, we can compute the mixing between the original Higgs eigenstates and the mass eigenstates to be 


$$
\begin{aligned}
\left(\begin{array}{c}
H_{+} \\
h \\
H
\end{array}\right)= & \left(\begin{array}{ccc}
1.0 & -3.6 \times 10^{-5} & 1.2 \times 10^{-7} \\
3.6 \times 10^{-5} & 1.0 & 0 \\
-1.2 \times 10^{-7} & 0 & 1.0
\end{array}\right) \\
& \times\left(\begin{array}{l}
h 1 \\
h 2 \\
h 3
\end{array}\right) .
\end{aligned}
$$

\section{B. Visible sector-induced breaking}

\section{Parameter scans}

Here, we implement a scan for visible-sector induced breaking, by scanning over parameters at the low-energy scale. As in the previous subsection, we insist on perturbativity for $\lambda_{S}$ and $g_{h}$ and take a maximum value of $m_{\gamma^{\prime}}$ of $40 \mathrm{GeV}$.

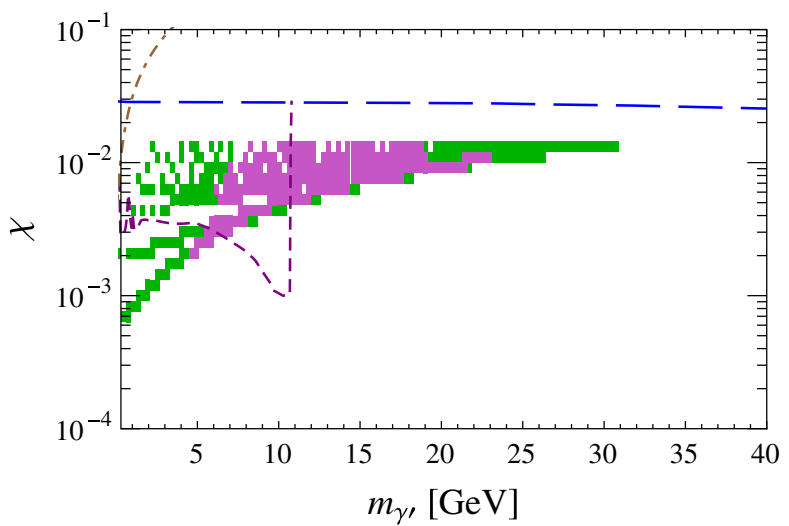

However, the soft supersymmetry breaking masses are chosen to be small, as may be induced in gauge mediation or sequestering of the hidden sector. Phenomenologically, then, the results of this subsection can be considered to be a detailed examination of the model of Ref. [20], but with a large gravitino mass and kinetic mixing respecting the relation (2.4).

As mentioned in Sec. II E, we can have either of two dark matter candidates, depending on the particular low-energy parameters: either the Majorana fermion $\tilde{o}_{1}$ or a Dirac fermion $\tilde{o}_{7}$. For both cases, we again use micrOMEGAs to compute the relic abundance and the scattering cross sections. The space of models in the kinetic-mixing-hiddenphoton mass plane (all points shown are in agreement with all direct-detection exclusions, including SIMPLE) is shown in Figs. 8 and 9; the color code is identical to the scatter plots for the toy model in Fig. 4, and the different experimental constraints are explained in Sec. III A and III C. Figure 8

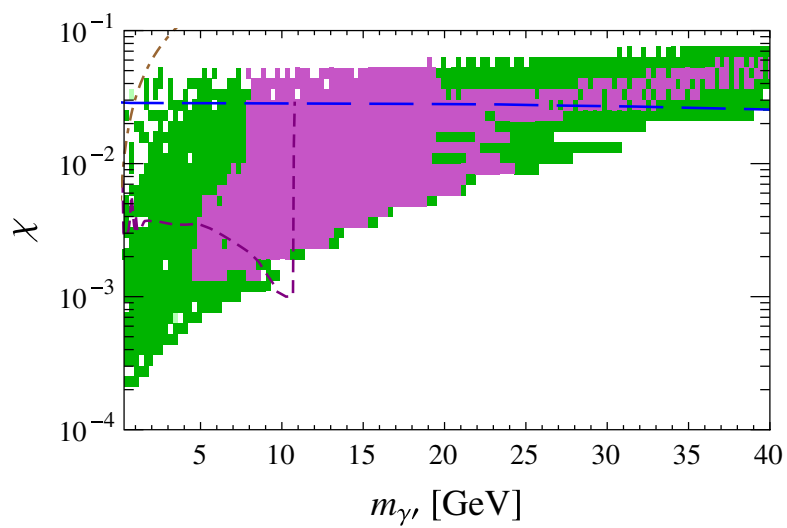

FIG. 8 (color online). Allowed space of models with visible-sector induced breaking as a function of the hidden photon mass and the magnitude of kinetic mixing, showing dark green areas (lighter grey) where the correct dark matter relic abundance can be found and light purple (darker grey) where the CoGeNT signal can be explained with a subdominant dark matter candidate. The lines represent the constraints from EWPT (long-dashed blue line), model dependent BABAR search (dashed dark) and muon $g-2$ (dashed-dotted). The SMH has been used, and all DD constraints are imposed including the SIMPLE exclusion limit. Left: $\kappa=1$, right: $0.1 \leq \kappa \leq 10$.
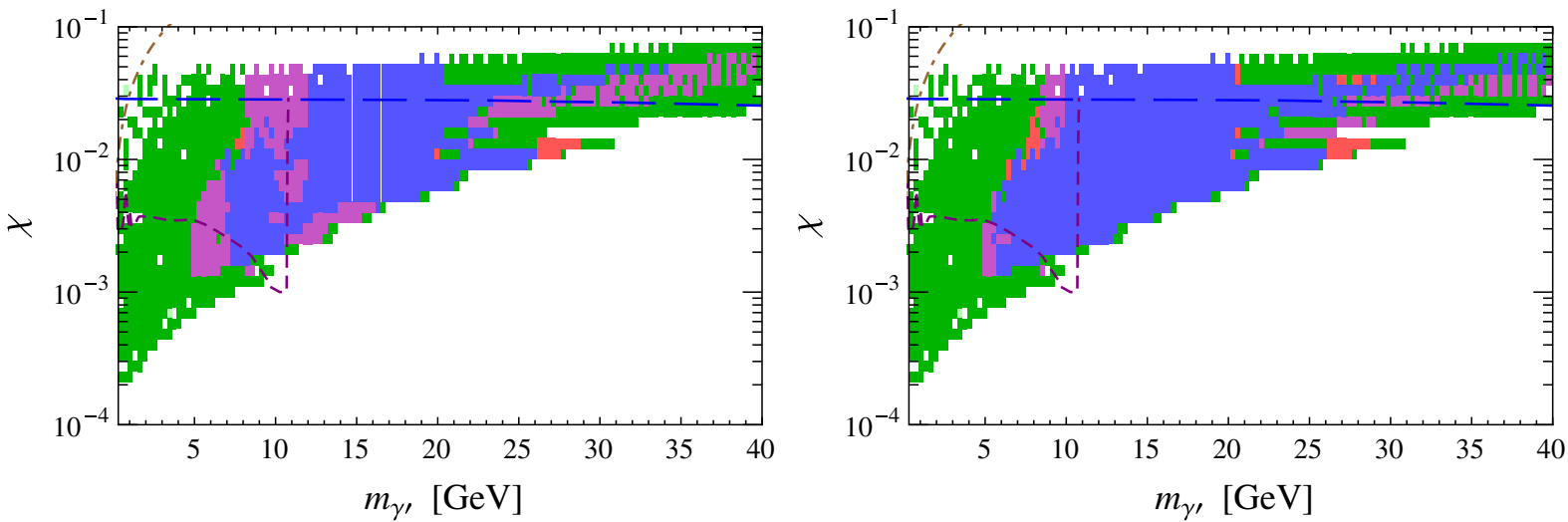

FIG. 9 (color online). Allowed space of models with the visible-sector induced breaking in function of the hidden photon mass and the magnitude of kinetic mixing, scanned over $0.1 \leq \kappa \leq 10$, showing different halo models. Left: Isothermal halo model; right: Einasto halo model. Here, the red region shows the space explaining the DAMA signal, the purple region explains the CoGeNT signal, and the blue one explains both DAMA and CoGeNT, all signal regions (darker grey shadings) having a subdominant dark matter density. All DD constraints including SIMPLE are imposed. The lines represent the constraints from EWPT (long-dashed blue line), model-dependent $B A B A R$ search (dashed dark) and muon $g-2$ (dashed-dotted). 
demonstrates the expansion in the parameter space by allowing a variation in $\kappa$; both $\kappa=1$ (left plot) and $0.1 \leq \kappa \leq 10$ (right plot) are shown for the SMH. The effect of changing the halo model is illustrated in Fig. 9. Depending on the halo model, we find subdominant DM explanations for DAMA and CoGeNT separately, as well as for both simultaneously, which are represented as light red, purple and blue regions, respectively. The two experiments can only be explained simultaneously for certain halo models other than the SMH.

The resulting parameter points in Figs. 8 and 9 show a very similar behavior to the toy model (see Fig. 4) as here the dark matter candidate can also be a Dirac fermion. The main difference is that the models here never permit annihilation via the $t$-channel diagram - since the dark matter particle can never be heavier than the hidden gauge boson. Therefore, the lower part of the plots is, in contrast to the toy model, empty as there it was filled by dark green points finding the correct relic abundance lying either just above the threshold for $t$-channel annihilation or on the $s$-channel

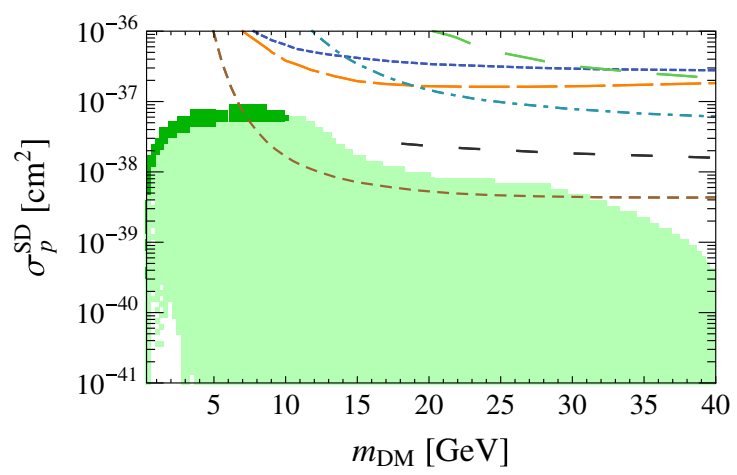

resonance (as we scan over the dark matter mass, these resonances move through the plot through different values of $m_{\gamma^{\prime}}$, and the whole range is covered). The coarser grid and small holes in the current scatter plots compared to the toy model arise from the fact that the parameter space cannot be scanned as continuously as for the toy model.

The spin-dependent and spin-independent scattering cross sections for the SMH are shown in Figs. 10 and 11, respectively. In both figures, the effect of including the SIMPLE limit is shown: points in yellow and orange indicate models whose SD scattering cross section on protons is excluded by SIMPLE while giving a subdominant and total DM abundance, respectively. The spin-dependent scattering cross sections in Fig. 10 are only appreciable when the Majorana fermion $\tilde{o}_{1}$ is the dark matter candidate. In Fig. 11 for SI scattering, there are two disjoint regions corresponding to whether the dark matter candidate is the Majorana fermion $\tilde{o}_{1}$ (lower region) or the Dirac fermion $\tilde{o}_{7}$ (upper region). As in the radiatively induced breaking case, the Majorana

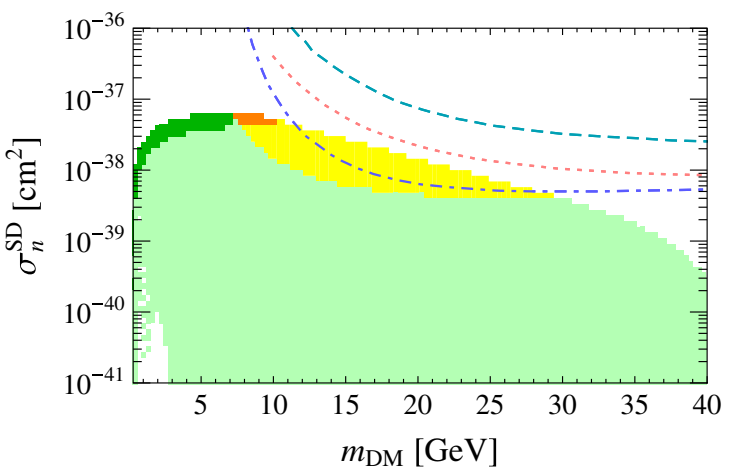

FIG. 10 (color online). Spin-dependent scattering cross sections on protons (left) and on neutrons (right) for visible-sector induced breaking together with the exclusion contours from the corresponding direct-detection experiments (the lowest lying, short-dashed brown line in the left plot is the SIMPLE limit; the other lines are as explained in Fig. 7, and in addition the long-dashed green line is the KIMS limit). Here, only the Majorana dark matter candidate $\tilde{o}_{1}$ is shown (the cross sections for the Dirac fermion $\tilde{o}_{7}$ are too small to appear). In the right plot, points in yellow and orange lie above the SIMPLE limit while giving a subdominant and total DM abundance respectively. The SMH has been used, and $0.1 \leq \kappa \leq 10$.
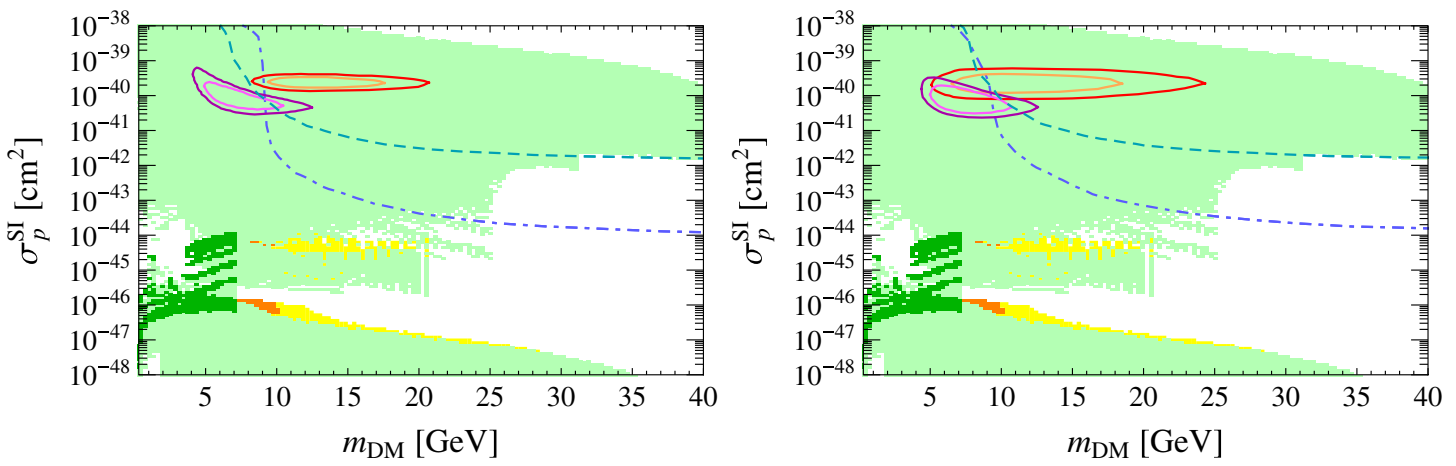

FIG. 11 (color online). Spin-independent scattering cross sections for visible-sector induced breaking using (left) the SMH and (right) the Einasto profile as given in Ref. [81] and $0.1 \leq \kappa \leq 10$. The signal contours from CoGeNT (purple ellipsoid more to the left) and DAMA (red ellipsoid more to the right) are shown, which overlap for the right-hand plot; the exclusion limits from CDMS and XENON100 are shown as dashed turquoise and dashed-dotted blue curves, respectively. The plot splits into two disjoint green areas: in the upper, the Dirac fermion $\tilde{o}_{7}$ is the dark matter candidate, while in the lower one, it is the Majorana fermion $\tilde{o}_{1}$. In both plots, points in yellow and orange lie above the SIMPLE limit while giving a subdominant and total DM abundance, respectively. 
fermion has a small spin-independent cross section of $10^{-47}$ to $10^{-45} \mathrm{~cm}^{2}$. In contrast to this, the spin-independent scattering cross section of the Dirac fermion $\tilde{o}_{7}$ is in the range of current direct-detection experiments and may explain the signals in CoGeNT and DAMA via a subdominant dark matter component. Hence, we present the effect of changing the halo model on the CoGeNT and DAMA regions in Fig. 11, showing that simultaneous explanations of both signals are possible and justify the blue regions in Fig. 9.

The Dirac fermion $\tilde{o}_{7}$ has almost no spin-dependent scattering on nuclei, so the SIMPLE exclusion limit only affects the lower regions in the plots for spin-independent scattering (Fig. 11) which correspond to the Majorana fermion, while the parameter regions that are interesting for spin-independent scattering experiments and can explain the DAMA and CoGeNT signals remain untouched. The SI scattering cross sections plotted are those for scattering on protons. As described above, the Dirac fermion's interaction through kinetic mixing couples almost exclusively to the charge of the nucleon, so its SI scattering on neutrons essentially vanishes. For the Majorana fermion $\tilde{o}_{1}$ on the other hand, spin-independent scattering on protons and neutrons is of roughly equal magnitude as it proceeds via the Higgs portal and squark exchange (as in the previous subsection), but the plots (Fig. 11) also show only the cross section on protons. As shown in Fig. 10, the spin-dependent scattering cross sections of the Majorana fermion DM candidate are also almost the same for protons and neutrons. However, the SIMPLE limit on the former (left plot) is slightly more constraining than the XENON 10 limit on the latter (right plot). In the case of the Dirac fermion dark matter, the spin-dependent scattering essentially vanishes both for protons and neutrons.

\section{Example model}

An example of a model that can explain the DAMA and CoGeNT signals (when we use the Einasto profile) is given in Table II where the spectrum is given. We take, at the low-energy scale, $\kappa=-10, \alpha_{h}\left(=\frac{g_{h}^{2}}{4 \pi}\right)=0.040$,
TABLE II. Hidden sector parameters and particle masses for an example sequestered model, yielding a dark matter density $\Omega_{\tilde{o}_{7}} h^{2}=1.7 \times 10^{-3}$ and rescaled direct detection cross section for scattering on protons $\sigma_{p}^{\mathrm{SI}}=1.0 \times 10^{-40} \mathrm{~cm}^{2}$.

\begin{tabular}{lc}
\hline \hline Low scale parameters & \\
\hline$\kappa$ & -10 \\
$\chi$ & -0.016 \\
$\alpha_{h}$ & 0.040 \\
$\alpha_{S}$ & 0.027 \\
$M_{\lambda}$ & $1.0(\mathrm{GeV})^{2}$ \\
$m_{H_{+}}^{2}$ & $1.0(\mathrm{GeV})^{2}$ \\
$m_{H_{-}}^{2}$ & $1.0(\mathrm{GeV})^{2}$ \\
$m_{S}^{2}$ & $1.0(\mathrm{GeV})^{2}$ \\
$A_{S}$ & 0.0 \\
\hline \hline \hline Particle & \\
\hline$\tilde{o}_{7}$ & Mass $(\mathrm{GeV})$ \\
$\tilde{o}_{1}$ & 6.4 \\
$\tilde{o}_{2}$ & 10.5 \\
$\gamma^{\prime}$ & 11.5 \\
$H_{+}$ & 11.0 \\
$H_{-}, S$ & 11.0 \\
\hline \hline
\end{tabular}

$\alpha_{S}\left(=\frac{\lambda_{S}^{2}}{4 \pi}\right)=0.027$ (giving $\chi=-0.016$ ) and the soft masses given by sequestered values $M_{\lambda}=m_{H_{+}}^{2}=m_{H_{+}}^{2}=m_{S}^{2}=$ $1 \mathrm{GeV}$, with the hidden A-term $A_{S}=0$. The dark matter candidate is necessarily then $\tilde{o}_{7}$, having a mass of $6.4 \mathrm{GeV}$ and yielding a density of $\Omega_{\tilde{o}_{7}} h^{2}=0.0021$. The cross section is almost entirely on protons, which, when rescaled to the dark matter density, yields an effective scattering cross section of $\sigma_{p}^{\mathrm{SI}}=1.0 \times 10^{-40} \mathrm{~cm}^{2}$. The hidden photon mass is $11 \mathrm{GeV}$, with width $3.7 \times 10^{-5} \mathrm{GeV}$, decaying mostly into light leptons and quarks. The hidden Higgs width is $2 \times 10^{-10} \mathrm{GeV}$, mostly decaying to charm and $\tau \mathrm{s}$.

For the SPS1b data point [100], the full neutralino mass matrix in the basis $\left(B_{0}, W_{0}, h_{u}^{0}, h_{d}^{0}, \tilde{\lambda}, \tilde{h}_{+}\right)$is

$$
\mathcal{M}_{\text {neutralino }}=\left(\begin{array}{cccccc}
166 & 0.00 & -2.73 & 43.8 & -0.02 & -0.18 \\
0.00 & 309 & 2.73 & -79.9 & 0.00 & 0.00 \\
-2.73 & 2.73 & 0.00 & -511 & 0.00 & 0.00 \\
43.8 & -79.90 & -511 & 0.00 & 0.00 & 0.00 \\
-0.02 & 0.00 & 0.00 & 0.00 & 1.00 & 11.0 \\
-0.18 & 0.00 & 0.00 & 0.00 & 11.0 & 0.00
\end{array}\right)
$$

with eigenmasses $10.5,11.5,164,298,516$, and $530 \mathrm{GeV}$. For the same data point, we can compute the mixing between the original Higgs eigenstates and the mass eigenstates to be

$$
\left(\begin{array}{c}
H_{+} \\
h \\
H
\end{array}\right)=\left(\begin{array}{ccc}
1.0 & -1.2 \times 10^{-3} & 3.9 \times 10^{-6} \\
1.2 \times 10^{-4} & 1.0 & 0 \\
-3.9 \times 10^{-6} & 0 & 1.0
\end{array}\right)\left(\begin{array}{l}
h 1 \\
h 2 \\
h 3
\end{array}\right)
$$




\section{CONCLUSIONS AND OUTLOOK}

We have presented what we believe to be the first detailed examination of the dark matter relic abundance and direct-detection cross sections of a complete stringinspired supersymmetric dark force model, emphasizing the natural supersymmetric relationship between kinetic mixing and the hidden gauge coupling. In particular, we have included running from high-energy gravitymediated boundary conditions and shown that interesting and viable models exist, in contrast to prior expectations. We have also examined the effect of neutralino mixing and the Higgs portal term, showing that the latter can contribute a small spin-independent cross section for Majorana fermion dark matter candidates. We examined the model in the cases of both radiative and visible-sector induced hidden gauge symmetry breaking and demonstrated the stark phenomenological contrasts between the two.

While the model can be used to explain the current dark matter signals observed by DAMA, CoGeNT and CRESST, this is not plausible in the case of radiativeinduced breaking relevant for gravity mediation, where our motivation was to show that simple dark sectors are not excluded - the hidden U(1) may instead be detected in fixed target experiments, particularly if the hidden photon cannot decay to hidden matter (as in the reasonably generic case when the dark matter particle has mass near that of the hidden photon). However, this is certainly plausible if the model were to be extended, for example, by allowing a supersymmetric mass for the singlet.
We hope that this work has paved the way for more detailed analysis of other supersymmetric dark sectors. In addition, there are several further possible avenues of work within the current model, for example, by including the full loop corrections to the effective potential, and constraints from indirect dark matter searches (which is a work in progress [89], although we believe them to be less stringent than the direct searches). It would also be interesting to compare the signal from CRESST with those from DAMA and CoGeNT in the context of these models.

\section{ACKNOWLEDGMENTS}

M. D. G. was supported by SFB Grant No. 676 and by the ERC advanced Grant No. 226371. He would like to thank Geneviève Belanger for helpful discussions and information about micrOMEGAs and James Wells for discussions about BBN. S. A. thanks Chiara Arina for helpful discussions on direct detection. All of the authors would like to thank Yann Mambrini for informative discussions about his related work, Andreas Goudelis for useful conversations about indirect detection and Jörg Jäckel and Saúl RamosSánchez for interesting conversations.

\section{APPENDIX A: RENORMALIZATION GROUP EQUATIONS}

Here, we present the two-loop renormalization group equations for the hidden-sector parameters $\alpha_{S} \equiv \lambda_{S}^{2} / 4 \pi$, $\alpha_{h}=g_{h}^{2} / 4 \pi, M_{\lambda}, m_{+}^{2}, m_{-}^{2}, m_{S}^{2}, A_{S}$. We define $t \equiv \log \mu$ :

$$
\begin{aligned}
\frac{d \alpha_{S}}{d t}= & \frac{1}{4 \pi}\left[2 \alpha_{S}\left(3 \alpha_{S}-4 \alpha_{h}\right)\right]+\frac{1}{(4 \pi)^{2}}\left[4 \alpha_{S}\left(-3 \alpha_{S}^{2}+2 \alpha_{S} \alpha_{h}+8 \alpha_{h}^{2}\right)\right] \frac{d \alpha_{h}}{d t}=\frac{1}{4 \pi}\left[4 \alpha_{h}^{2}\right]+\frac{1}{(4 \pi)^{2}}\left[8 \alpha_{h}^{2}\left(2 \alpha_{h}-\alpha_{S}\right)\right] \\
\frac{d M_{\lambda}}{d t}= & \frac{1}{4 \pi}\left[4 \alpha_{h} M_{\lambda}\right]+\frac{1}{(4 \pi)^{2}}\left[8 \alpha_{h} M_{\lambda}\left(4 \alpha_{h}^{2}-\alpha_{S}\right)+8 \alpha_{h} \alpha_{S} A_{S}\right] \\
\frac{d A_{S}}{d t}= & \frac{1}{4 \pi}\left[6 \alpha_{S} A_{S}+8 \alpha_{h} M_{\lambda}\right]+\frac{1}{(4 \pi)^{2}}\left[8 A_{S}\left(\alpha_{h} \alpha_{S}-3 \alpha_{S}^{2}\right)-8 \alpha_{h} M_{\lambda}\left(\alpha_{S}+8 \alpha_{h}\right)\right] \\
\frac{d m_{S}^{2}}{d t}= & \frac{1}{4 \pi}\left[2 \alpha_{S}\left(m_{S}^{2}+m_{+}^{2}+m_{-}^{2}+A_{S}^{2}\right)\right]+\frac{1}{(4 \pi)^{2}}\left[8 \alpha_{S}\left(\alpha_{h}-\alpha_{S}^{2}\right)\left(m_{S}^{2}+m_{+}^{2}+m_{-}^{2}+2 A_{S}^{2}\right)+8 \alpha_{h} \alpha_{S}\left(2 M_{\lambda}^{2}-2 M_{\lambda} A_{S}-A_{S}^{2}\right)\right] \\
\frac{d m_{ \pm}^{2}}{d t}= & \frac{1}{4 \pi}\left[2 \alpha_{S}\left(m_{S}^{2}+m_{+}^{2}+m_{-}^{2}+A_{S}^{2}\right)-8 M_{\lambda}^{2} \alpha_{h} \pm 2 \alpha_{h}\left(m_{+}^{2}-m_{-}^{2}\right)\right]+\frac{1}{(4 \pi)^{2}}\left[-8 \alpha_{S}^{2}\left(m_{S}^{2}+m_{+}^{2}+m_{-}^{2}+2 A_{S}^{2}\right)\right. \\
& \left.+96 \alpha_{h}^{2} M_{\lambda}^{2}+8 \alpha_{h}^{2}\left(m_{+}^{2}+m_{-}^{2}\right) \pm 4 \alpha\left(2 \alpha_{h}-\alpha_{S}\right)\left(m_{+}^{2}-m_{-}^{2}\right)\right] .
\end{aligned}
$$

The reader may be surprised to see that the kinetic mixing or the visible-sector parameters are absent, but this is perfectly correct to two-loop order. This is because the kinetic mixing is a one-loop quantity [as can be seen from the canonical Lagrangian (2.1)], and we maintain the normalization of the gauge field strengths throughout the running to be

$$
\mathcal{L} \supset \int d^{2} \theta\left(\frac{1}{4} B^{\alpha} B_{\alpha}+\frac{1}{4} X^{\alpha} X_{\alpha}-\frac{\chi}{2} B^{\alpha} X_{\alpha}\right),
$$

i.e., we do not diagonalize the gauge fields. Until we arrive at low energies, the hypercharge and hidden U(1) are massless, and so there is a continuous choice of basis for the fields. However, we have two key assumptions: first, we are assuming that the kinetic mixing is generated by a high-energy theory, above the scale at which we begin the running, and also that there are no states charged under both U(1)s. If we were to diagonalize the U(1)s throughout the RGE trajectory, we would introduce millicharges, and so to return to this 
basis at low energies, we would necessarily have to undo the transformation, affecting all of the parameters. Fortunately, maintaining in this basis with these assumptions greatly simplifies the RGEs, and it is easy to see that $\chi$ only appears as $\chi^{2}$. The largest effect is then induced through the visible sector terms; the leading corrections to the above RGEs are given by [20]

$$
\delta\left(\frac{d m_{ \pm}^{2}}{d t}\right)=-\frac{8 \chi^{2} \alpha_{h}}{4 \pi}\left|M_{1}\right|^{2} \quad \delta\left(\frac{d A_{S}}{d t}\right)=-\frac{8 \chi^{2} \alpha_{h}}{4 \pi} M_{1},
$$

where $M_{1}$ is the Bino mass. When we recall Eq. (2.4), we see that this correction becomes

$$
-\frac{8 \chi^{2} \alpha_{h}}{4 \pi}\left|M_{1}\right|^{2} \rightarrow-\frac{8 \kappa^{2} \alpha_{h}^{2} \alpha_{Y}}{(4 \pi)^{3}}\left|M_{1}\right|^{2},
$$

i.e., it is a three-loop effect that can be safely neglected in the models we consider in this paper-particularly since we assume gravity-mediated generation of soft masses; these effects might be important (i.e., providing a leading but still very small contribution) if we were to take some of the them to be zero, for example, in little gauge mediation when the singlet and gaugino masses vanish.

\section{APPENDIX B: SPECTRUM OF THE MODEL}

In this Appendix, we present the details of the low-energy features of the model $W=\lambda_{S} S H_{+} H_{-}$.
Once supersymmetry and R-symmetry is broken, the potential is

$$
\begin{aligned}
V= & \left|\lambda_{S}\right|^{2}\left(\left|S H_{+}\right|^{2}+\left|S H_{-}\right|^{2}+\left|H_{+} H_{-}\right|^{2}\right) \\
& +\frac{g_{h}^{2}}{2}\left(\left|H_{+}\right|^{2}-\left|H_{-}\right|^{2}-\xi\right)^{2}+m_{+}^{2}\left|H_{+}\right|^{2}+m_{-}^{2}\left|H_{-}\right|^{2} \\
& +m_{S}^{2}|S|^{2}+\left(\lambda_{S} A_{S} S H_{+} H_{-}+\text {c.c. }\right) .
\end{aligned}
$$

We are assuming that no $\mu, B_{\mu}$ terms are generated; these would introduce new scales into the theory. Although they could conceivably be generated by a Giudice-Masiero mechanism in analogy with the visible sector, we shall neglect this possibility.

\section{Scalars}

Defining $\Delta \equiv \sqrt{\lambda_{S}^{2} \xi-m_{+}^{2} \lambda_{S}^{2} / g_{h}^{2}}$, we have mass matrices in the $\left(H_{+}, H_{+}^{\dagger}\right)$ basis of

$$
\frac{1}{2}\left(H_{+}^{\dagger} H_{+}\right)\left(\begin{array}{cc}
g^{2} \Delta^{2} / \lambda_{S}^{2} & g_{h}^{2} \Delta^{2} / \lambda_{S}^{2} \\
g_{h}^{2} \Delta^{2} / \lambda_{S}^{2} & g_{h}^{2} \Delta^{2} / \lambda_{S}^{2}
\end{array}\right)\left(\begin{array}{c}
H_{+} \\
H_{+}^{\dagger}
\end{array}\right),
$$

which implies masses for the two components of $\sqrt{2} g_{h} \Delta / \lambda_{S}, 0$ at this level. The "massless" mode is the Goldstone boson that becomes the longitudinal component of the massive gauge field. The $\left(H_{-}, H_{-}^{\dagger}, S, S^{\dagger}\right)$ system is more complicated; we find a mass matrix of

$$
\frac{1}{2}\left(H_{-}^{\dagger} H_{-} S^{\dagger} S\right)\left(\begin{array}{cccc}
\Delta^{2}+m_{+}^{2}+m_{-}^{2} & 0 & 0 & A_{S}^{\dagger} \Delta \\
0 & \Delta^{2}+m_{+}^{2}+m_{-}^{2} & A_{S} \Delta & 0 \\
0 & A_{S}^{\dagger} \Delta & \Delta^{2}+m_{S}^{2} & 0 \\
A_{S} \Delta & 0 & 0 & \Delta^{2}+m_{S}^{2}
\end{array}\right)\left(\begin{array}{c}
H_{-} \\
H_{-}^{\dagger} \\
S \\
S^{\dagger}
\end{array}\right) .
$$

The above theory for nonzero $\xi$ has a minimum at $\left\langle H_{+}\right\rangle=$ $\Delta / \lambda_{S}$ provided that $\Delta$ is real, and

$$
\begin{aligned}
& 0 \leq m_{-}^{2}+m_{+}^{2}+m_{S}^{2}+2 \Delta^{2} \\
& 0 \leq\left(m_{-}^{2}+m_{+}^{2}+\Delta^{2}\right)\left(m_{S}^{2}+\Delta^{2}\right)-\left|A_{S}\right|^{2} \Delta^{2} .
\end{aligned}
$$

In the case $A_{S}=0, m_{+}^{2}=m_{-}^{2}<0$, this translates simply to the condition that $\lambda_{S}^{2} \geq 2 g_{h}^{2}$.

\section{Fermions and neutralino mixing}

The fermion mass matrix in the basis $\left(\tilde{\lambda}, \tilde{h}_{+}, \tilde{h}_{-}, \tilde{s}\right)$ (neglecting the kinetic mixing of the gaugino with the neutralino) is given by

$$
\mathcal{M}_{f}=\left(\begin{array}{cccc}
M_{\lambda} & m_{\gamma^{\prime}} & 0 & 0 \\
m_{\gamma^{\prime}} & 0 & 0 & 0 \\
0 & 0 & 0 & \Delta \\
0 & 0 & \Delta & 0
\end{array}\right)
$$

However, to properly compute the dark matter density, we should take mixing of the fermions with the neutralino into account. The fields $\tilde{h}_{-}, \tilde{s}$ form a Dirac fermion that does not mix with any other fields. There will, however, be kinetic mixing of the Bino with the hidden gaugino and possibly mass mixing; writing these fields before the mixing as, respectively, $\tilde{b}, \tilde{\lambda}$ we can define

$$
\tilde{b}=\frac{1}{c_{\epsilon}} b \quad \tilde{\lambda}=\lambda-t_{\epsilon} b
$$

which then allows us to write the full neutralino mass matrix in the basis $\left(B_{0}, W_{0}, h_{u}^{0}, h_{d}^{0}, \tilde{\lambda}, \tilde{h}_{+}\right)$, including the standard Majorana masses for Bino and Wino $M_{1,2}$ but also a potential explicit mass mixing term $\mathcal{L} \supset-m_{X} \tilde{b} \tilde{\lambda}$ as 


$$
\left(\begin{array}{cccccc}
\frac{1}{c_{\epsilon}^{2}}\left[M_{1}-s_{\epsilon} m_{X}+s_{\epsilon}^{2} M_{\lambda}\right] & 0 & -M_{Z} s_{W} c_{\beta} / c_{\epsilon} & M_{Z} s_{W} s_{\beta} / c_{\epsilon} & \frac{1}{c_{\epsilon}}\left[m_{X}-s_{\epsilon} M_{\lambda}\right] & -m_{\gamma}^{\prime} t_{\epsilon} \\
0 & M_{2} & M_{Z} c_{W} c_{\beta} & -M_{Z} c_{W} s_{\beta} & 0 & 0 \\
-M_{Z} s_{W} c_{\beta} / c_{\epsilon} & M_{Z} c_{W} c_{\beta} & 0 & -\tilde{\mu} & 0 & 0 \\
M_{Z} s_{W} s_{\beta} / c_{\epsilon} & -M_{Z} c_{W} s_{\beta} & -\tilde{\mu} & 0 & 0 & 0 \\
\frac{1}{c_{\epsilon}}\left[m_{X}-s_{\epsilon} M_{\lambda}\right] & 0 & 0 & 0 & M_{\lambda} & m_{\gamma}^{\prime} \\
-m_{\gamma}^{\prime} t_{\epsilon} & 0 & 0 & 0 & m_{\gamma}^{\prime} & 0
\end{array}\right) .
$$

\section{APPENDIX C: KINETIC AND MASS MIXING}

Here, we review the diagonalization of the gauge fields and the subsequent coupling of the physical gauge bosons to matter fields.

Consider the Lagrangian coupling the currents $j_{B}, j_{W}$ and $j_{X}$ to the respective unrotated gauge bosons $\tilde{B}_{\mu}, \tilde{W}_{\mu}, \tilde{X}_{\mu}$ (corresponding to hypercharge, weak and hidden gauge bosons):

$$
\begin{aligned}
\mathcal{L}= & -\frac{1}{4} \tilde{B}_{\mu \nu} \tilde{B}^{\mu \nu}-\frac{1}{4} \tilde{X}_{\mu \nu} \tilde{X}^{\mu \nu}+\frac{\chi}{2} \tilde{B}_{\mu \nu} \tilde{X}^{\mu \nu}-\frac{1}{4} \tilde{W}_{\mu \nu} \tilde{W}^{\mu \nu}+\frac{1}{2} \tilde{m}^{2} \tilde{X}_{\mu} \tilde{X}^{\mu}+\frac{1}{8} v^{2}\left(g_{Y} \tilde{B}_{\mu}-g_{2} \tilde{W}_{\mu}\right)^{2} \\
& +g_{Y} j_{B}^{\mu} \tilde{B}_{\mu}+g_{2} j_{W}^{\mu} \tilde{W}_{\mu+} g_{h} j_{X}^{\mu} \tilde{X}_{\mu} \\
= & -\frac{1}{4} B_{\mu \nu} B^{\mu \nu}-\frac{1}{4} X_{\mu \nu} X^{\mu \nu}-\frac{1}{4} \tilde{W}_{\mu \nu} \tilde{W}^{\mu \nu}+g_{2} j_{W}^{\mu} \tilde{W}_{\mu}+g_{Y} j_{B}^{\mu} B_{\mu}+\frac{1}{\sqrt{1-\chi^{2}}}\left(g_{h} j_{X}^{\mu}+\chi g_{Y} j_{B}^{\mu}\right) X_{\mu} \\
& +\frac{\tilde{m}^{2}}{1-\chi^{2}} \frac{1}{2} X_{\mu} X^{\mu}+\frac{1}{8} v^{2}\left(g_{Y} B_{\mu}+\frac{g_{Y} \chi}{\sqrt{1-\chi^{2}}} X_{\mu}-g_{2} \tilde{W}_{\mu}\right)^{2} .
\end{aligned}
$$

Then, we make the transformation

$$
\begin{aligned}
\tilde{W}_{\mu} & \equiv s_{W} A_{\mu}+c_{W}\left(c_{\phi} Z_{\mu}+s_{\phi} \gamma^{\prime \mu}\right) \\
\tilde{B}_{\mu} & \equiv c_{W} A_{\mu}-s_{W}\left(c_{\phi} Z_{\mu}+s_{\phi} \gamma^{\prime \mu}\right)+\frac{\chi}{\sqrt{1-\chi^{2}}}\left(c_{\phi} \gamma^{\prime \mu}-s_{\phi} Z_{\mu}\right) \\
& =c_{W} A_{\mu}-\left(s_{W} c_{\phi}+\frac{\chi}{\sqrt{1-\chi^{2}}} s_{\phi}\right) Z_{\mu}+\left(\frac{c_{\phi} \chi}{\sqrt{1-\chi^{2}}}-s_{W} s_{\phi}\right) \gamma^{\prime \mu} \\
\tilde{X}_{\mu} & \equiv \frac{1}{\sqrt{1-\chi^{2}}}\left(-s_{\phi} Z_{\mu}+c_{\phi} \gamma_{\mu}^{\prime}\right),
\end{aligned}
$$

where $c_{W}, s_{W}$ are the usual cosine and sine of the weak mixing angle, respectively, and $c_{\phi}, s_{\phi}$ are the cosine and sine of an angle $\phi$ to be determined below so that

$$
\begin{aligned}
\mathcal{L} \supset & -\frac{1}{4} F_{\mu \nu} F^{\mu \nu}+m_{\gamma^{\prime}}^{2} \frac{1}{2} \gamma_{\mu}^{\prime}\left(\gamma^{\prime}\right)^{\mu}+M_{Z}^{2} \frac{1}{2} Z_{\mu} Z^{\mu}+e A_{\mu}\left[j_{W}^{\mu}+j_{B}^{\mu}\right] \\
& +Z_{\mu}\left[g_{2} c_{W} c_{\phi} j_{W}^{\mu}-\left(s_{W} c_{\phi}+\frac{\chi^{s} s_{\phi}}{\sqrt{1-\chi^{2}}}\right) g_{Y} j_{B}^{\mu}-\frac{g_{X} s_{\phi}}{\sqrt{1-\chi^{2}}} j_{X}^{\mu}\right] \\
& +\gamma_{\mu}^{\prime}\left[g_{2} c_{W} s_{\phi} j_{W}^{\mu}+\left(\frac{c_{\phi} \chi}{\sqrt{1-\chi^{2}}}-s_{W} s_{\phi}\right) g_{Y} j_{B}^{\mu}+\frac{g_{X} c_{\phi}}{\sqrt{1-\chi^{2}}} j_{X}^{\mu}\right] .
\end{aligned}
$$

We find, defining $x \equiv \tilde{m}^{2} / M_{Z}^{2}\left(\simeq m_{\gamma^{\prime}}^{2} / M_{Z}^{2}\right)$,

$$
\tan 2 \phi=-\frac{2 s_{W} s_{\epsilon} c_{\epsilon}}{c_{\epsilon}^{2}-s_{W}^{2} s_{\epsilon}^{2}-x} \quad \sin \phi=\frac{s_{W} \chi}{1-x}+\cdots,
$$

where $s_{\epsilon}=-\chi, c_{\epsilon}=\sqrt{1-\chi^{2}}$ were defined in the text.

In terms of these, we have the eigenvalues where $m_{+}$corresponds to the physical $Z$ mass and $m_{-}$to the physical hidden photon mass:

$$
\begin{aligned}
& m_{ \pm}^{2}=\frac{1}{2}\left[m^{2}+\frac{M_{Z}^{2}}{\cos ^{2} \alpha} \pm \sqrt{\left(\frac{M_{Z}^{2}}{\cos ^{2} \alpha}+m^{2}\right)^{2}-4 m^{2} M_{Z}^{2}}\right] \quad m_{+}^{2}=M_{Z}^{2}\left[1+\frac{s_{W}^{2} \chi^{2}}{1-x}+\cdots\right] \\
& m_{-}^{2}=\tilde{m}^{2}\left[1+\frac{\left(1-s_{W}^{2}-x\right) \chi^{2}}{1-x}+\cdots\right] .
\end{aligned}
$$

Thus, the masses are only shifted at order $\chi^{2}$. 
Note that we can also write

$$
m_{+}^{2}=M_{Z}^{2}\left(c_{\phi}-s_{W} t_{\epsilon} s_{\phi}\right)^{2}+\frac{\tilde{m}^{2}}{c_{\epsilon}^{2}} s_{\phi}^{2} \quad m_{-}^{2}=M_{Z}^{2}\left(s_{\phi}+s_{W} t_{\epsilon} c_{\phi}\right)^{2}+\frac{\tilde{m}^{2}}{c_{\epsilon}^{2}} c_{\phi}^{2},
$$

and, defining $\hat{x} \equiv m_{-}^{2} / m_{+}^{2} \approx x$, we have

$$
\tan \phi=\frac{-(1-\hat{x}) \pm \sqrt{(1-\hat{x})^{2}-4 s_{W}^{2} t_{\epsilon}^{2} x}}{2 s_{W} t_{\epsilon} \hat{x}} .
$$

\section{APPENDIX D: GOLDSTONE BOSON MIXING}

Here, we consider the effect of the mixing on the Goldstone bosons that are eaten and what happens to the other fields. Assuming that the visible sector is the MSSM with neutral Goldstone boson $G^{0}$, and taking the hidden sector to be broken by a single complex scalar $C=\frac{1}{\sqrt{2}}\left(C_{R}+i C_{I}\right)$, we have

$$
\begin{aligned}
Ł & \supset\left|\partial_{\mu} C-i g_{h} \tilde{X}_{\mu} C\right|^{2}+\left|\partial_{\mu} H_{u}^{0}-\frac{i}{2}\left(g_{Y} B_{\mu}-g_{2} W_{\mu}^{0}\right) H_{u}^{0}\right|^{2}+\left|\partial_{\mu} H_{d}^{0}+\frac{i}{2}\left(g_{Y} B_{\mu}-g_{2} W_{\mu}^{0}\right) H_{d}^{0}\right|^{2}+V_{\mathrm{hid}}(C)+V_{\mathrm{vis}}(H) \\
= & \frac{1}{2}\left(\partial_{\mu} C_{I}-m_{h} \tilde{X}_{\mu}\right)^{2}+\frac{1}{2}\left(\partial_{\mu} C_{R}\right)^{2}+\frac{1}{2}\left[\partial_{\mu}\left(c_{\beta} H_{3}-s_{\beta} G^{0}\right)-\frac{1}{2}\left(g_{Y} B_{\mu}-g_{2} W_{\mu}^{0}\right) v s_{\beta}\right]^{2} \\
& +\frac{1}{2}\left[\partial_{\mu}\left(s_{\beta} H_{3}+c_{\beta} G^{0}\right)+\frac{1}{2}\left(g_{Y} B_{\mu}-g_{2} W_{\mu}^{0}\right) v c_{\beta}\right]^{2}+\cdots \\
& \supset \frac{1}{2}\left(\partial_{\mu} C_{I}-m_{h} \tilde{X}_{\mu}\right)^{2}+\frac{1}{2}\left[\partial_{\mu} G^{0}-\frac{e v}{s_{2 W}}\left(c_{W} W_{\mu}^{0}-s_{W} B_{\mu}\right)\right]^{2}+\cdots .
\end{aligned}
$$

Clearly, the masses of the neutral Higgs (both visible and hidden) are unaffected by the mixing, but the pseudoscalar will be. Using Eq. (C2) and

$$
c_{W} W_{\mu}^{0}-s_{W} B_{\mu}=\left(c_{\phi}-s_{W} t_{\epsilon} s_{\phi}\right) Z_{\mu}+\left(s_{\phi}+s_{W} t_{\epsilon} c_{\phi}\right) \gamma_{\mu}^{\prime}
$$

we obtain

$$
\begin{aligned}
\mathcal{L} & \supset-m_{h} \partial_{\mu} C_{I} \frac{1}{c_{\epsilon}}\left(-s_{\phi} Z_{\mu}+c_{\phi} \gamma_{\mu}^{\prime}\right)-M_{Z^{0}} \partial_{\mu} G^{0}\left(\left(c_{\phi}-s_{W} t_{\epsilon} s_{\phi}\right) Z_{\mu}+\left(s_{\phi}+s_{W} t_{\epsilon} c_{\phi}\right) \gamma_{\mu}^{\prime}\right) \\
& \supset-Z_{\mu}\left(-m_{h} \frac{s_{\phi}}{c_{\epsilon}} \partial_{\mu} C_{I}+M_{Z^{0}}\left(c_{\phi}-s_{W} t_{\epsilon} s_{\phi}\right) \partial_{\mu} G^{0}\right)-\gamma_{\mu}^{\prime}\left(m_{h} \frac{c_{\phi}}{c_{\epsilon}} \partial_{\mu} C_{I}+M_{Z^{0}}\left(s_{\phi}+s_{W} t_{\epsilon} c_{\phi}\right) \partial_{\mu} G^{0}\right) .
\end{aligned}
$$

Thus,

$$
m_{+} G_{Z}=M_{Z^{0}}\left(c_{\phi}-s_{W} t_{\epsilon} s_{\phi}\right) G^{0}-m_{h} \frac{s_{\phi}}{c_{\epsilon}} C_{I} \quad m_{-} G_{\gamma^{\prime}}=M_{Z^{0}}\left(s_{\phi}+s_{W} t_{\epsilon} c_{\phi}\right) G^{0}+m_{h} \frac{c_{\phi}}{c_{\epsilon}} C_{I}
$$

and

$$
\begin{aligned}
G^{0} & =\frac{c_{\epsilon}}{M_{Z^{0}} m_{h}}\left[\frac{m_{h} c_{\phi} m_{+}}{c_{\epsilon}} G_{Z}+\frac{m_{h} s_{\phi} m_{-}}{c_{\epsilon}} G_{\gamma^{\prime}}\right]=\frac{1}{M_{Z^{0}}}\left[c_{\phi} m_{+} G_{Z}+s_{\phi} m_{-} G_{\gamma^{\prime}}\right] \\
C_{I} & =\frac{c_{\epsilon}}{m_{h}}\left[-\left(s_{\phi}+s_{W} t_{\epsilon} c_{\phi}\right) m_{+} G_{Z}+\left(c_{\phi}-s_{W} t_{\epsilon} s_{\phi}\right) m_{-} G_{\gamma^{\prime}}\right] .
\end{aligned}
$$

We can also write this as

$$
G^{0}=\cos \psi G_{Z}+\sin \psi G_{\gamma^{\prime}} \quad C_{I}=-\sin \psi G_{Z}+\cos \psi G_{\gamma^{\prime}} \quad \tan \psi=(\tan \phi) \frac{m_{-}}{m_{+}}=\frac{\left(s_{\phi}+s_{W} t_{\epsilon} c_{\phi}\right) m_{+}}{\left(c_{\phi}-s_{W} t_{\epsilon} s_{\phi}\right) m_{-}}
$$

\section{APPENDIX E: HIGGS PORTAL MIXING}

With the Lagrangian density

$$
\mathcal{L} \supset \int d^{2} \theta\left(\frac{1}{4} B^{\alpha} B_{\alpha}+\frac{1}{4} X^{\alpha} X_{\alpha}-\frac{\chi}{2} B^{\alpha} X_{\alpha}\right)+\text { c.c., }
$$

we need the $D$-term mixing. We write $\hat{D}_{Y} \equiv-g_{Y} \sum_{\phi} \phi^{\dagger} \hat{Y} \phi, \hat{D}_{X} \equiv-g_{h} \sum_{\phi} \phi^{\dagger} \hat{Q}_{X} \phi$ as the $D$-terms in the absence of mixing; then, we have

$$
\mathcal{L} \supset \frac{1}{2} D_{Y}^{2}+\frac{1}{2} D_{X}^{2}-\chi D_{Y} D_{X}-D_{Y} \hat{D}_{Y}-D_{X} \hat{D}_{X}
$$


which leads to

$D_{X}=\frac{1}{1-\chi^{2}}\left(\hat{D}_{X}+\chi \hat{D}_{Y}\right) \quad D_{Y}=\frac{1}{1-\chi^{2}}\left(\hat{D}_{Y}+\chi \hat{D}_{X}\right)$

and thus

$$
\begin{aligned}
V & \rightarrow \frac{1}{2} \frac{1}{1-\chi^{2}}\left[\hat{D}_{X^{2}}+\hat{D}_{Y^{2}}+2 \chi \hat{D}_{X} \hat{D}_{Y}\right], \\
V_{\text {portal }} & \equiv \frac{\chi}{1-\chi^{2}} \hat{D}_{X} \hat{D}_{Y} .
\end{aligned}
$$

The relevant part of the potential for us involves the Higgses; we can write the portal term as

$$
\begin{aligned}
V_{\text {portal }}= & \frac{\chi}{1-\chi^{2}} g_{Y} g_{h}\left(\left|H_{+}\right|^{2}-\left|H_{-}\right|^{2}\right)\left(\frac{1}{2}\left|H_{u}\right|^{2}-\frac{1}{2}\left|H_{d}\right|^{2}\right) \\
= & \frac{\chi g_{Y} g_{h}}{1-\chi^{2}}\left(\left|H_{+}\right|^{2}-\left|H_{-}\right|^{2}\right) \\
& \times\left[\frac{1}{2}\left|H_{u}^{+}\right|^{2}+\frac{1}{2}\left(\frac{1}{\sqrt{2}} v \sin \beta+H_{u}^{0}\right)^{2}\right. \\
& \left.-\frac{1}{2}\left|H_{d}^{-}\right|^{2}-\frac{1}{2}\left(\frac{1}{\sqrt{2}} v \cos \beta+H_{d}^{0}\right)^{2}\right]
\end{aligned}
$$

Immediately, we can extract the effective Fayet-Iliopoulos term:

$$
\begin{aligned}
V_{\mathrm{FI}} & =\frac{g_{h}^{2}}{2}\left(\left|H_{+}\right|^{2}-\left|H_{-}\right|^{2}-\xi\right)^{2}=\frac{1}{2}\left(\hat{D}_{h}+\xi / g_{h}\right)^{2} \\
\xi & \approx\left(\chi / g_{h}\right)\left\langle\hat{D}_{Y\rangle}=\left(\chi / g_{h}\right) g_{Y} \frac{v^{2}}{4} \cos 2 \beta .\right.
\end{aligned}
$$

However, we can also extract the Higgs mass mixing. Writing

$$
\begin{aligned}
H_{+} & =\frac{v_{+}}{\sqrt{2}}+\frac{1}{\sqrt{2}}\left(x_{R}+i x_{I}\right) \\
H_{u}^{0} & =\frac{1}{\sqrt{2}}\left[s_{\beta} v+h_{u}^{0}+i\left(c_{\beta} A-s_{\beta} G^{0}\right)\right] \\
H_{d}^{0} & =\frac{1}{\sqrt{2}}\left[c_{\beta} v+h_{d}^{0}+i\left(s_{\beta} A+c_{\beta} G^{0}\right)\right] \\
\left(\begin{array}{c}
h_{d}^{0} \\
h_{u}^{0}
\end{array}\right) & =\left(\begin{array}{cc}
\cos \alpha & -\sin \alpha \\
\sin \alpha & \cos \alpha
\end{array}\right)\left(\begin{array}{l}
H \\
h
\end{array}\right)
\end{aligned}
$$

and using the standard shorthand $c_{\beta} \equiv \cos \beta, c_{\alpha} \equiv \cos \alpha$, $c_{\alpha+\beta} \equiv \cos (\alpha+\beta)$, etc., we have

$$
\begin{aligned}
V & \supset-\frac{t_{\epsilon}}{c_{\epsilon}} g_{Y} g_{h}\left|\frac{v_{+}}{\sqrt{2}}+\frac{1}{\sqrt{2}}\left(x_{R}+i x_{I}\right)\right|^{2}\left[\frac{1}{2}\left|\frac{1}{\sqrt{2}}\left[s_{\beta} v+h_{u}^{0}+i\left(c_{\beta} A-s_{\beta} G^{0}\right)\right]\right|^{2}-\frac{1}{2}\left|\frac{1}{\sqrt{2}}\left[c_{\beta} v+h_{d}^{0}+i\left(s_{\beta} A+c_{\beta} G^{0}\right)\right]\right|^{2}\right] \\
& \supset-\frac{t_{\epsilon}}{c_{\epsilon}} g_{Y} g_{h} \frac{1}{2} v_{+} v x_{R}\left[s_{\alpha+\beta} h-c_{\alpha+\beta} H\right] \equiv \frac{1}{2} M_{m}^{2} x_{R}\left[-s_{\alpha+\beta} h+c_{\alpha+\beta} H\right] \\
M_{m}^{2} & \equiv \frac{t_{\epsilon}}{c_{\epsilon}} g_{Y} g_{h} v \frac{\sqrt{2} \Delta}{\lambda_{S}}=\frac{t_{\epsilon}}{c_{\epsilon}} 2 M_{Z} s_{W} m_{\gamma^{\prime}} \approx-2 \chi s_{W} M_{Z} m_{\gamma^{\prime}} .
\end{aligned}
$$

So then we must redefine our Higgses: the mass mixing matrix in the basis $\left(x_{+}, h, H\right)$ is

$$
\mathcal{M}_{\text {Higgs }}^{2}=\left(\begin{array}{ccc}
m_{+}^{2} & -s_{\alpha+\beta} M_{m}^{2} & c_{\alpha+\beta} M_{m}^{2} \\
-s_{\alpha+\beta} M_{m}^{2} & m_{h}^{2} & 0 \\
c_{\alpha+\beta} M_{m}^{2} & 0 & m_{H}^{2}
\end{array}\right) .
$$

To first order in $M_{m}^{2} / m_{h, H}^{2}$, this is diagonalized via

$$
\left(\begin{array}{c}
x_{+} \\
h \\
H
\end{array}\right)=\left(\begin{array}{ccc}
1 & -\frac{s_{\alpha+\beta} M_{m}^{2}}{m_{h}^{2}-m_{+}^{2}} & \frac{c_{\alpha+\beta} M_{m}^{2}}{m_{H}^{2}-m_{+}^{2}} \\
\frac{s_{\alpha+\beta} M_{m}^{2}}{m_{h}^{2}-m_{+}^{2}} & 1 & 0 \\
-\frac{c_{\alpha+\beta} M_{m}^{2}}{m_{H}^{2}-m_{+}^{2}} & 0 & 1
\end{array}\right)\left(\begin{array}{c}
x_{R}^{\prime} \\
h^{\prime} \\
H^{\prime}
\end{array}\right) .
$$

Since we often find $m_{H}^{2} \gg m_{h}^{2}$, however, the above will usually reduce to mixing between the hidden and lightest Higgs. In this case, we can approximate

$$
\begin{aligned}
\left(\begin{array}{c}
x_{R} \\
h
\end{array}\right) & \approx\left(\begin{array}{cc}
1 & u \\
-u & 1
\end{array}\right)\left(\begin{array}{c}
x_{R}^{\prime} \\
h^{\prime}
\end{array}\right) \\
u & \approx \frac{-s_{\alpha+\beta} M_{m}^{2}}{m_{h}^{2}-m_{+}^{2}} \approx s_{\alpha+\beta} 2 \chi s_{W} \frac{M_{Z} m_{\gamma^{\prime}}}{m_{h}^{2}} .
\end{aligned}
$$

Thus, very roughly, $u \sim \chi s_{W^{\prime}} m_{\gamma^{\prime}} / m_{h}$ for large $\tan \beta$.

\section{Spin-independent nucleon cross sections}

Here, we would like to estimate the cross sections for our Majorana hidden fermion $\tilde{O}_{1}=\left(\begin{array}{c}\tilde{o}_{1} \\ \tilde{\tilde{o}}_{1}\end{array}\right)$ on nucleons that take place via the Higgs portal term. The Higgs portal leads to an effective four-point interaction

$$
\mathcal{L} \supset f_{N}\left(\overline{\tilde{O}}_{1} \tilde{O}_{1} \bar{N} N\right),
$$

and we can consider different $f_{p}, f_{n}$ for protons and neutrons, respectively. Consider that the dark matter 
particle is a Majorana combination of $\tilde{\lambda}$ and $\tilde{h}_{+}$fermions, $\tilde{o}_{1} \approx \cos \theta_{1} \tilde{h}_{+}+\sin \theta_{1} \tilde{\lambda}$. The coupling to the hidden Higgs is via the kinetic vertex

$$
\begin{aligned}
\mathcal{L} & \supset-\sqrt{2} g_{h} H_{+}^{*}\left(\tilde{h}_{+} \tilde{\lambda}\right)+\text { c.c. } \\
& \supset-g_{h} \cos \theta_{1} \sin \theta_{1} x_{R}\left[\left(\tilde{o}_{1} \tilde{o}_{1}\right)+\left(\overline{\tilde{o}}_{1} \overline{\tilde{o}}_{1}\right)\right] \\
& \supset-g_{h} \cos \theta_{1} \sin \theta_{1} x_{R} \overline{\tilde{O}}_{1} \tilde{O}_{1},
\end{aligned}
$$

where now $\tilde{O}_{1}$ is in Dirac form, $\tilde{O}_{1}=\left(\begin{array}{c}\tilde{o}_{1} \\ \tilde{\tilde{o}}_{1}\end{array}\right)$. Now let us write the coupling of the MSSM Higgs to nucleons as $-a_{N} h \bar{N} N$.

$$
\begin{aligned}
\mathcal{L} \supset & -g_{h} \cos \theta_{1} \sin \theta_{1}\left[x_{R}^{\prime}+u h^{\prime}\right] \overline{\tilde{O}}_{1} \tilde{O}_{1} \\
& -a_{N}\left[-u x_{R}^{\prime}+h^{\prime}\right] \bar{N} N-\frac{1}{2} m_{1}^{2}\left(x_{R}^{\prime}\right)^{2}-\frac{1}{2} m_{2}^{2}\left(h^{\prime}\right)^{2} \\
\rightarrow & u a_{N} \cos \theta_{1} \sin \theta_{1}\left[\frac{1}{m_{1}^{2}}-\frac{1}{m_{2}^{2}}\right]\left(\overline{\tilde{O}}_{1} \tilde{O}_{1} \bar{N} N\right) \\
\approx & a_{N} \cos \theta_{1} \sin \theta_{1} s_{\alpha+\beta} 2 \chi s_{W} \frac{M_{Z} m_{\gamma^{\prime}}}{m_{+}^{2} m_{h}^{2}}\left(\overline{\tilde{O}}_{1} \tilde{O}_{1} \bar{N} N\right) \\
\rightarrow & f_{N} \approx a_{N} \sin 2 \theta_{1} s_{\alpha+\beta} \chi s_{W} \frac{M_{Z} m_{\gamma^{\prime}}}{m_{+}^{2} m_{h}^{2}} .
\end{aligned}
$$

So then, our direct-detection amplitude should be approximately given by the coefficient above.

The coupling of the MSSM Higgs to nucleons is determined by its coupling to quarks. These come from

$$
\begin{aligned}
\mathcal{L} & \supset-Y_{U} H_{U} q u-Y_{D} H_{D} q d \\
& \supset-\frac{c_{\alpha}}{v s_{\beta}} h\left(m_{U} \bar{u} u\right)-\frac{s_{\alpha}}{v c_{\beta}} h\left(m_{D} \bar{d} d\right) .
\end{aligned}
$$

Then, we use that [101]

$$
\begin{aligned}
\left\langle N\left|m_{q} \bar{q} q\right| N\right\rangle & =m_{n} f_{T q}^{(N)} \\
\left\langle N\left|m_{Q} \bar{Q} Q\right| N\right\rangle_{Q=c, b, t} & =\frac{2}{27} m_{N}\left[1-\sum_{q=u, d, s} f_{T q}^{(N)}\right] \\
& \equiv \frac{2}{27} m_{N}\left[1-\tilde{F}^{(N)}\right]
\end{aligned}
$$

to give

$$
\begin{aligned}
a_{N}= & \frac{c_{\alpha}}{v s_{\beta}} m_{N}\left[\frac{4}{27}(1-\tilde{F})+f_{T u}^{(N)}\right] \\
& +\frac{s_{\alpha}}{v c_{\beta}} m_{N}\left[\frac{2}{27}(1-\tilde{F})+f_{T d}^{(N)}+f_{T s}^{(N)}\right] .
\end{aligned}
$$

If we consider large $\tan \beta$ with $\beta=\pi / 2-\delta, \alpha \approx-\delta$, we have $s_{\alpha} \approx-\delta, c_{\alpha} \approx 1, c_{\beta} \approx \delta, s_{\beta} \approx 1$ and so $a_{N} \approx$ $\frac{m_{N}}{v}\left[\frac{2}{27}(1-\tilde{F})+f_{T u}^{(N)}-f_{T d}^{(N)}-f_{T s}^{(N)}\right]$. Let us then simply define

$$
a_{N} \equiv \frac{m_{N}}{v} \hat{f}^{(N)} .
$$

There are large uncertainties in the value of $\hat{f}^{(N)}$, however, it is approximately equal for protons and neutrons and varies from about 0.03 to 0.44 . We can then take an approximate value for the amplitude for a Majorana fermion scattering on nucleons to be

$$
f_{N} \approx \sin 2 \theta_{1} s_{\alpha+\beta} \chi s_{W} \frac{M_{Z} m_{\gamma^{\prime}}}{m_{+}^{2} m_{h}^{2}} \frac{m_{N}}{v} \hat{f}^{(N)} .
$$

Taking the large $\tan \beta$ values, $m_{h}=115 \mathrm{GeV}$ and $\hat{f}^{(N)} \sim$ 0.1 , we obtain

$f_{N} \approx 3 \times 10^{-9}(\mathrm{GeV})^{-2} \times\left(\sin 2 \theta_{1}\right)\left(\frac{\chi s_{W}}{0.001}\right)\left(\frac{\mathrm{GeV}}{m_{\gamma^{\prime}}}\right)$.

This is clearly a very small effect. This translates into a cross section for scattering on a single nucleon of

$$
\begin{aligned}
\sigma_{N}^{\text {SI,portal }}= & \frac{4 m_{\tilde{o} 1}^{2} m_{N}^{2}}{\pi\left(m_{N}+m_{\tilde{o} 1}\right)^{2}} f_{N}^{2} \\
\approx & 2 \times 10^{-45} \mathrm{~cm}^{2} \times\left(\frac{m_{\tilde{o} 1}^{2}}{\left(m_{N}+m_{\tilde{o} 1}\right)^{2}}\right)\left(\sin 2 \theta_{1}\right)^{2} \\
& \times\left(\frac{\chi s_{W}}{0.001}\right)^{2}\left(\frac{\mathrm{GeV}}{m_{\gamma^{\prime}}}\right)^{2} .
\end{aligned}
$$

This corresponds well to the values that we find in the plots (see Fig. 11).

The above will be supplemented by contributions from $s$-channel squark exchange. Very roughly for these, we have effective four-point interactions of the Majorana fermion with quarks with coupling $f_{q} \sim \frac{g^{2}}{M_{Q}^{2}}\left|U_{\tilde{b} \tilde{o}_{1}}\right|^{2}$, where $\tilde{b}=\sum_{n} U_{b \tilde{o}_{n}} \tilde{o}_{n}$ is the Bino, which mixes most strongly with the lightest hidden state. By considering the mass mixing matrix in Sec. B 2, we can conclude that (in the absence of direct mass mixing) the mixing is simply $\sim \chi M_{\lambda}$, so we have $U_{\tilde{b} \tilde{o}_{1}} \sim \chi$, and thus

$$
f_{N} \sim \frac{g_{Y}^{2}}{M_{Q}^{2}} \chi^{2} \sum_{q} \frac{m_{N}}{m_{q}} f_{T q}^{N},
$$

and thus

$$
\begin{aligned}
\sigma_{N}^{\text {SD,Squark }} \sim & 10^{-48} \mathrm{~cm}^{2} \times\left(\frac{m_{\tilde{\sigma}_{1}}^{2}}{\left(m_{N}+m_{\tilde{o}_{1}}\right)^{2}}\right)\left(\frac{s_{W} \chi}{0.001}\right)^{4} \\
& \times\left(\frac{100 \mathrm{GeV}}{M_{\tilde{Q}}}\right)^{4} .
\end{aligned}
$$


[1] D. Feldman, B. Kors, and P. Nath, Phys. Rev. D 75, 023503 (2007).

[2] M. Pospelov, A. Ritz, and M. B. Voloshin, Phys. Lett. B 662, 53 (2008).

[3] N. Arkani-Hamed, D. P. Finkbeiner, T. R. Slatyer, and N. Weiner, Phys. Rev. D 79, 015014 (2009).

[4] M. Pospelov and A. Ritz, Phys. Lett. B 671, 391 (2009).

[5] N. Arkani-Hamed and N. Weiner, J. High Energy Phys. 12 (2008) 104

[6] O. Adriani et al. (PAMELA Collaboration), Nature (London) 458, 607 (2009).

[7] J. Chang, J. Adams, H. Ahn, G. Bashindzhagyan, M. Christl et al., Nature (London) 456, 362 (2008).

[8] A. A. Abdo et al. (Fermi LAT Collaboration), Phys. Rev. Lett. 102, 181101 (2009).

[9] R. Bernabei et al. (DAMA Collaboration), Eur. Phys. J. C 56, 333 (2008).

[10] C. Aalseth et al. (CoGeNT Collaboration), Phys. Rev. Lett. 106, 131301 (2011).

[11] C. Aalseth, P. Barbeau, J. Colaresi, J. Collar, J. Diaz Leon et al., Phys. Rev. Lett. 107, 141301 (2011).

[12] G. Angloher, M. Bauer, I. Bavykina, A. Bento, C. Bucci et al., Eur. Phys. J. C 72, 1971 (2012).

[13] J. Collar, arXiv:1106.0653.

[14] J. Collar, arXiv:1103.3481.

[15] E. Aprile et al. (XENON100 Collaboration), Phys. Rev. Lett. 107, 131302 (2011).

[16] Z. Ahmed (CDMS-II Collaboration), Phys. Rev. Lett. 106, 131302 (2011).

[17] E. J. Chun and J.-C. Park, J. Cosmol. Astropart. Phys. 02 (2009) 026.

[18] C. Cheung, J. T. Ruderman, L.-T. Wang, and I. Yavin, Phys. Rev. D 80, 035008 (2009).

[19] A. Katz and R. Sundrum, J. High Energy Phys. 06 (2009) 003.

[20] D. E. Morrissey, D. Poland, and K. M. Zurek, J. High Energy Phys. 07 (2009) 050.

[21] T. Cohen, D. J. Phalen, A. Pierce, and K. M. Zurek, Phys. Rev. D 82, 056001 (2010).

[22] Z. Kang, T. Li, T. Liu, C. Tong, and J. M. Yang, J. Cosmol. Astropart. Phys. 01 (2011) 028.

[23] S. A. Abel, M. D. Goodsell, J. Jaeckel, V. V. Khoze, and A. Ringwald, J. High Energy Phys. 07 (2008) 124.

[24] M. Goodsell, arXiv:0912.4206.

[25] M. Goodsell, J. Jaeckel, J. Redondo, and A. Ringwald, J. High Energy Phys. 11 (2009) 027.

[26] M. Goodsell and A. Ringwald, Fortschr. Phys. 58, 716 (2010).

[27] J. J. Heckman and C. Vafa, Phys. Rev. D 83, 026006 (2011).

[28] M. Bullimore, J. P. Conlon, and L. T. Witkowski, J. High Energy Phys. 11 (2010) 142.

[29] M. Cicoli, M. Goodsell, J. Jaeckel, and A. Ringwald, J. High Energy Phys. 07 (2011) 114.

[30] M. Williams, C. Burgess, A. Maharana, and F. Quevedo, J. High Energy Phys. 08 (2011) 106.

[31] J. J. Heckman and S.-J. Rey, J. High Energy Phys. 06 (2011) 120.

[32] K. R. Dienes, C.F. Kolda, and J. March-Russell, Nucl. Phys. B492, 104 (1997).
[33] A. Lukas and K. S. Stelle, J. High Energy Phys. 01 (2000) 010.

[34] R. Blumenhagen, G. Honecker, and T. Weigand, J. High Energy Phys. 06 (2005) 020.

[35] M. Goodsell, S. Ramos-Sanchez, and A. Ringwald, J. High Energy Phys. 01 (2012) 021.

[36] S. A. Abel and B.W. Schofield, Nucl. Phys. B685, 150 (2004).

[37] D. Lust and S. Stieberger, Fortschr. Phys. 55, 427 (2007).

[38] S. A. Abel, J. Jaeckel, V. V. Khoze, and A. Ringwald, Phys. Lett. B 666, 66 (2008).

[39] K. Benakli and M. D. Goodsell, Nucl. Phys. B830, 315 (2010).

[40] F. Gmeiner and G. Honecker, Nucl. Phys. B829, 225 (2010).

[41] R. Foot, H. Lew, and R. Volkas, Phys. Lett. B 272, 67 (1991).

[42] R. Schabinger and J. D. Wells, Phys. Rev. D 72, 093007 (2005).

[43] D. Feldman, Z. Liu, and P. Nath, Phys. Rev. D 75, 115001 (2007).

[44] C.P. Burgess, J.P. Conlon, L.-Y. Hung, C. H. Kom, A. Maharana, and F. Quevedo, J. High Energy Phys. 07 (2008) 073.

[45] A. Hook, E. Izaguirre, and J. G. Wacker, Adv. High Energy Phys. 2011, 859762 (2011).

[46] E. Dudas, Y. Mambrini, S. Pokorski, and A. Romagnoni, J. High Energy Phys. 08 (2009) 014.

[47] Y. Mambrini, J. Cosmol. Astropart. Phys. 12 (2009) 005.

[48] Y. Mambrini, Proc. Sci. QFTHEP2010 (2010) 027.

[49] Y. Mambrini, J. Cosmol. Astropart. Phys. 09 (2010) 022.

[50] Y. Mambrini, J. Cosmol. Astropart. Phys. 07 (2011) 009.

[51] G. Belanger, F. Boudjema, A. Pukhov, and A. Semenov, Comput. Phys. Commun. 149, 103 (2002).

[52] G. Belanger, F. Boudjema, A. Pukhov, and A. Semenov, Comput. Phys. Commun. 176, 367 (2007).

[53] G. Belanger, F. Boudjema, A. Pukhov, and A. Semenov, Comput. Phys. Commun. 177, 894 (2007).

[54] G. Belanger, F. Boudjema, A. Pukhov, and A. Semenov, Comput. Phys. Commun. 180, 747 (2009).

[55] G. Belanger, F. Boudjema, A. Pukhov, and A. Semenov, Nuovo Cimento Soc. Ital. Fis. C 033N2, 111 (2010).

[56] R. H. Cyburt, J. R. Ellis, B. D. Fields, and K. A. Olive, Phys. Rev. D 67, 103521 (2003).

[57] J. Jaeckel and A. Ringwald, Annu. Rev. Nucl. Part. Sci. 60, 405 (2010).

[58] M. Pospelov, Phys. Rev. D 80, 095002 (2009).

[59] R. Essig, P. Schuster, and N. Toro, Phys. Rev. D 80, 015003 (2009).

[60] E. Riordan, M. Krasny, K. Lang, P. De Barbaro, A. Bodek et al., Phys. Rev. Lett. 59, 755 (1987).

[61] J. D. Bjorken, S. Ecklund, W. R. Nelson, A. Abashian, C. Church, B. Lu, L.W. Mo, T. A. Nunamaker, and P. Rassmann, Phys. Rev. D 38, 3375 (1988).

[62] A. Bross, M. Crisler, S. H. Pordes, J. Volk, S. Errede, and J. Wrbanek, Phys. Rev. Lett. 67, 2942 (1991).

[63] J.D. Bjorken, R. Essig, P. Schuster, and N. Toro, Phys. Rev. D 80, 075018 (2009).

[64] M. Davier and H. Nguyen Ngoc, Phys. Lett. B 229, 150 (1989).

[65] S. Andreas, arXiv:1110.2636. 
[66] H. Merkel et al. (A1 Collaboration) Phys. Rev. Lett. 106, 251802 (2011).

[67] S. Abrahamyan et al. (APEX Collaboration), Phys. Rev. Lett. 107, 191804 (2011).

[68] J. Blumlein and J. Brunner, Phys. Lett. B 701, 155 (2011).

[69] F. Archilli, D. Babusci, D. Badoni, I. Balwierz, G. Bencivenni et al., Phys. Lett. B 706, 251 (2012).

[70] R. Essig, P. Schuster, N. Toro, and B. Wojtsekhowski, J. High Energy Phys. 02 (2011) 009.

[71] M. Freytsis, G. Ovanesyan, and J. Thaler, J. High Energy Phys. 01 (2010) 111.

[72] Heavy Photon Search (HPS) Collaboration, https:// confluence.slac.stanford.edu/display/hpsg/.

[73] E. Komatsu et al. (WMAP Collaboration), Astrophys. J. Suppl. Ser. 192, 18 (2011).

[74] F. Giuliani, Phys. Rev. Lett. 95, 101301 (2005).

[75] J. L. Feng, J. Kumar, D. Marfatia, and D. Sanford, Phys. Lett. B 703, 124 (2011).

[76] M. T. Frandsen, F. Kahlhoefer, J. March-Russell, C. McCabe, M. McCullough, and K. Schmidt-Hoberg, Phys. Rev. D 84, 041301 (2011).

[77] C. McCabe, Phys. Rev. D 84, 043525 (2011).

[78] M. T. Frandsen, F. Kahlhoefer, S. Sarkar, and K. SchmidtHoberg, J. High Energy Phys. 09 (2011) 128.

[79] X. Gao, Z. Kang, and T. Li, arXiv:1107.3529.

[80] J. M. Cline and A. R. Frey, Phys. Rev. D 84, 075003 (2011).

[81] C. Arina, J. Hamann, and Y. Y. Wong, J. Cosmol. Astropart. Phys. 09 (2011) 022.

[82] P. Ullio, M. Kamionkowski, and P. Vogel, J. High Energy Phys. 07 (2001) 044.
[83] R. Kappl and M. W. Winkler, Nucl. Phys. B850, 505 (2011).

[84] E. Behnke, J. Behnke, S. Brice, D. Broemmelsiek, J. Collar et al., Phys. Rev. Lett. 106, 021303 (2011).

[85] M. Felizardo et al. (SIMPLE Collaboration), Phys. Rev. Lett. 108, 201302 (2012).

[86] J. Collar, arXiv:1106.3559.

[87] SIMPLE Collaboration, arXiv:1107.1515.

[88] S. Desai et al. (Super-Kamiokande Collaboration), Phys. Rev. D 70, 083523 (2004).

[89] S. Andreas, M. D. Goodsell, A. Goudelis, and A. Ringwald (work in progress).

[90] J. Angle, E. Aprile, F. Arneodo, L. Baudis, A. Bernstein et al. (XENON10 Collaboration), Phys. Rev. Lett. 101, 091301 (2008).

[91] D. Y. Akimov, H. Araujo, E. Barnes, V. Belov, A. Bewick et al., Phys. Lett. B 709, 14 (2012).

[92] Z. Ahmend et al. (CDMS Collaboration) Phys. Rev. Lett. 102, 011301 (2009).

[93] Z. Ahmed et al. (CDMS-II Collaboration) Science 327, 1619 (2010).

[94] A. Semenov, arXiv:hep-ph/9608488.

[95] A. Semenov, Comput. Phys. Commun. 115, 124 (1998).

[96] A. Semenov, arXiv:hep-ph/0208011.

[97] A. Semenov, Comput. Phys. Commun. 180, 431 (2009).

[98] A. Semenov, arXiv:1005.1909.

[99] B. Allanach, Comput. Phys. Commun. 143, 305 (2002).

[100] B. Allanach, M. Battaglia, G. Blair, M. S. Carena, A. De Roeck et al., Eur. Phys. J. C 25, 113 (2002).

[101] G. Jungman, M. Kamionkowski, and K. Griest, Phys. Rep. 267, 195 (1996). 